
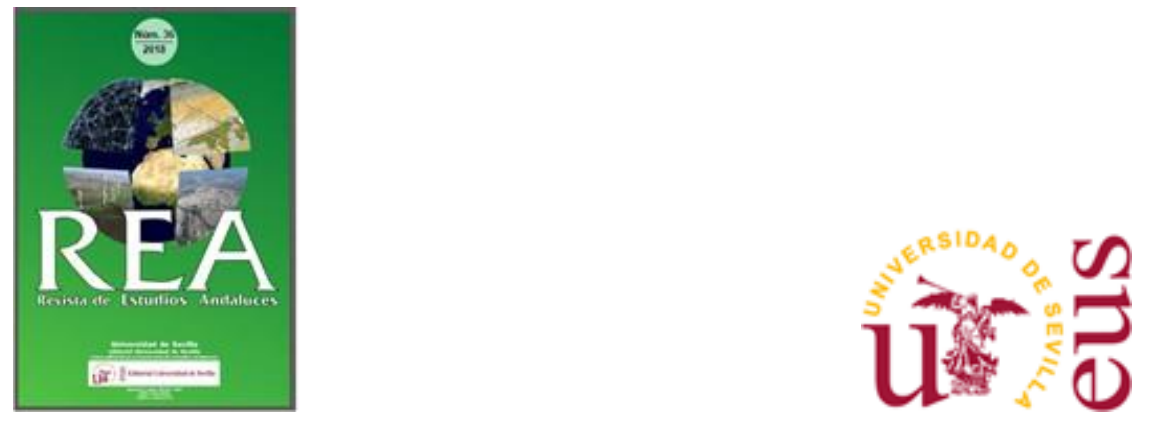

\author{
Revista de Estudios Andaluces (REA) \\ e-ISSN: 2340-2776.
}

REA Núm. 36 (2018). http://dx.doi.org/10.12795/rea.2018.i36

\title{
La Evaluación de las Políticas de Regeneración Urbana. Propuesta Metodológica para la Delimitación de Áreas Experimentales y Equivalentes
}

\section{Evaluating Urban Regeneration Policies. Methodological Proposal to Delimitate Experimental and Equivalent Urban Areas}

\author{
José Manuel Betanzos-Martín \\ Autonomía Sur, SCA \\ betani81@gmail.com \\ Antonio Echaves-García \\ Universidad Pablo de Olavide \\ aechgar@upo.es \\ Rafael Moya-Alonso \\ Comunidad de Madrid \\ rafael.moya2@educa.madrid.org \\ Clemente J. Navarro-Yáñez \\ Universidad Pablo de Olavide \\ cnavyan@upo.es
}

\begin{abstract}
Formato de cita / Citation: Betanzos-Martín, José Manuel, Echaves-García, Antonio, Moya-Alonso, Rafael, Navarro-Yáñez, Clemente J. (2018). La Evaluación de las Políticas de Regeneración Urbana. Propuesta Metodológica para la Delimitación de Áreas Experimentales y Equivalentes. Revista de Estudios Andaluces, núm. 36, 147-177.
\end{abstract}

http://dx.doi.org/10.12795/rea.2018.i36.07

Enlace artículo/to link to this article: http://dx.doi.org/10.12795/rea.2018.i36.07

(c) $($ ()) $\Theta$ 


\title{
La Evaluación de las Políticas de Regeneración Urbana. Propuesta Metodológica para la Delimitación de Áreas Experimentales y Equivalentes $^{1}$
}

\section{Evaluating Urban Regeneration Policies. Methodological Proposal to Delimitate Experimental and Equivalent Urban Areas}

\author{
José Manuel Betanzos-Martín \\ Autonomía Sur, SCA \\ betani81@gmail.com \\ Antonio Echaves-García \\ Universidad Pablo de Olavide \\ aechgar@upo.es \\ Rafael Moya-Alonso \\ Comunidad de Madrid \\ rafael.moya2@educa.madrid.org \\ Clemente J. Navarro-Yáñez \\ Universidad Pablo de Olavide \\ cnavyan@upo.es
}

Recibido: 26 de febrero, 2018

Revisado: 13 de junio, 2018

Aceptado: 16 de junio, 2018

\section{Resumen}

El presente artículo presenta una propuesta metodológica para la delimitación de áreas territoriales aplicando una estrategia de corte cuasi-experimental, comparando las áreas en las que se ha desarrollado algún proyecto de regeneración urbana llamadas "áreas experimentales" con otras similares en las que no se ha desarrollado ningún proyecto, denominadas "áreas equivalentes". Esta metodología es el paso previo necesario para el análisis de los efectos de los programas de regeneración urbana, que permitirá posteriormente comparar la evolución en el tiempo de pares semejantes entre sí (área experimental y área equivalente). En primer lugar, se explica el objetivo que subyace a la delimitación de estas áreas territoriales. En segundo lugar, se describe la metodología utilizada para seleccionar las áreas equivalentes. En tercer lugar, se presentan los resultados, esto es, la delimitación de áreas experimentales y equivalentes. Por último, mediante

\footnotetext{
${ }^{1}$ Este artículo es resultado del trabajo desarrollado en el marco del proyecto Regeneración Urbana y Cohesión Social (GGI30011/DIY) financiado por la convocatoria de I+D+I de la Consejería de Fomento y Vivienda de la Junta de Andalucía y FEDER. El proyecto fue desarrollado en el Centro de Sociología y Políticas Locales-The Urban Governance Lab, de la Universidad Pablo de Olavide (www.upo.es/cspl). Los autores agradecen a los miembros del Área de Vivienda y Rehabilitación de la Consejería de Fomento y Vivienda su apoyo y asesoramiento en el desarrollo del proyecto.

Revista de Estudios Andaluces, núm. 36 (2018) pp. 147-177. e-ISSN: 2340-2776

http://dx.doi.org/10.12795/rea.2018.i36.07

(c) (i) $\odot$

cc) Esta obra se distribuye con la licencia Creative Commons Reconocimiento-NoComercial-SinObraDerivada 4.0 Internacional (CC BY-NC-ND 4.0.)
} 
procedimientos estadísticos concretos, análisis de varianza o ANOVA de un factor, se validan los resultados obtenidos. El trabajo se centra en las ciudades andaluzas de más de 100 mil habitantes en las que se han ejecutado proyectos de regeneración urbana.

Palabras clave: políticas de regeneración urbana, programas de regeneración urbana, desigualdad urbana, barrios vulnerables, ciudades andaluzas.

\begin{abstract}
This article analyses a methodological proposal for the delimitation of territorial areas applying a quasi-experimental strategy, comparing areas where projects have been developed based in urban regeneration called "experimental areas" with similar ones without any project, called "equivalent areas". This methodology is a necessary previous step to analyze the effect of programs about urban regeneration, which will allow subsequently to compare the evolution of similar pairs among themselves (experimental and equivalent areas). Firstly, we explain the underlying objective of delimitation in these territorial areas. Secondly, the methodology used to select the equivalent areas is described. Thirdly, the results are presented, it means, the delimitation of experimental and equivalent areas. Finally, through means of specific statistical procedures, analysis of variance or ANOVA of a factor, the obtained results are validated. The work is focused on Andalusian cities with a population over 100.000 inhabitants in which urban regeneration projects have been implemented.
\end{abstract}

Keywords: urban regeneration policies, urban regeneration programs, urban inequality, distressed urban areas, Andalusian cities. 


\section{INTRODUCCIÓN}

En buena medida, la evaluación de impacto de las políticas de regeneración urbana supone analizar si el proyecto desarrollado en las áreas que han sido objeto de intervención ha supuesto una mejora en la calidad de vida existente en ellas. Esto supone que un paso necesario para ese tipo de ejercicio evaluativo consiste en delimitar territorialmente el área intervenida, pero también otras similares con las que pueda compararse para evaluar de forma más certera el impacto de la intervención desarrollada.

En este marco, el presente trabajo plantea una propuesta metodológica para la delimitación de áreas territoriales que son, o pueden ser, objeto de intervención mediante políticas públicas urbanas de diversa índole (de carácter residencial, social o económico), como paso previo para el análisis de los efectos que los programas de regeneración urbana tienen sobre el territorio. En concreto, aquí se consideran tres programas desarrollados en Andalucía: el programa de Áreas de Rehabilitación de Barrios (en adelante ARB) ejecutado por la Consejería de Fomento y Vivienda, centrado en aspectos residenciales y la promoción de vivienda pública y rehabilitación; URBAN, programa financiado por la Unión Europea que desarrolla actuaciones de índole económica, social, medioambiental y física; y finalmente las Zonas con Necesidades de Transformación Social (en adelante ZNTS), desarrollado por la Consejería de Igualdad, y que se centra, fundamentalmente en actuaciones de carácter social y de desarrollo cívico².

Todos los programas analizados suponen la delimitación de un área territorial en la que se desarrolla un proyecto de actuación específico. El área de intervención supone, pues, el ámbito en el que se espera que se produzca los efectos del proyecto, y por acumulación, de los programas en su conjunto. Cabe suponer que el desarrollo de un proceso de regeneración urbana redunde en ciertos efectos beneficiosos en el área territorial en el que se aplica, tanto a los aspectos referidos al entorno y la vivienda, como a otros de índole socioeconómica, que deberían evidenciarse a través de indicadores que midan estos aspectos en momentos previos y posteriores al de la intervención desarrollada.

Otra forma de enfrentarse a esta cuestión podría ser, quizás, la siguiente pregunta: ¿qué hubiese pasado si no se hubiese realizado el proyecto de regeneración en esa área territorial? Evidentemente, la comparación del área consigo misma antes y después de la intervención da cuenta del cambio habido, pero no permite responder adecuadamente a esa cuestión. Para ello sería necesario, al menos, comparar los cambios producidos en otra área similar en el momento previo o al iniciarse la intervención. Cabría esperar que los efectos de esta última se evidenciasen porque las pautas de cambios en el entorno y socioeconómicos fuesen diferentes entre el área territorial objeto de intervención y la que, siendo similar, no ha sido objeto de intervención.

A los dos tipos de áreas definidas se denominará en adelante 'área experimental', aquella en la que se ejecuta un proyecto, y 'área equivalente', aquella que es similar en la primera pero donde no se

\footnotetext{
${ }^{2}$ Más detalles sobre estos proyectos pueden consultarse en la web del proyecto RUCOSA:

http://www.centrosociologia.geographica.gs/

Revista de Estudios Andaluces, núm. 36 (2018) pp.147-177. e-ISSN: 2340-2776

http://dx.doi.org/10.12795/rea.2018.i36.07
}

(c) (i) $\ominus$

cc) Esta obra se distribuye con la licencia Creative Commons Reconocimiento-NoComercial-SinObraDerivada 4.0 Internacional (CC BY-NC-ND 4.0.) 
ejecuta ningún tipo de proyecto, sea del mismo programa o de los otros que se están analizando. Aquí se presenta la metodología utilizada para delimitar las áreas equivalentes para los proyectos ejecutados en las ciudades andaluzas de más de 100 mil habitantes, en concreto: Algeciras, Almería, Cádiz, Córdoba, Dos Hermanas, Granada, Huelva, Jaén, Jerez de la Frontera, Málaga y Sevilla.

\section{2. ¿POR QUÉ COMPARAR ÁREAS EXPERIMENTALES Y EQUIVALENTES? LA SELECCIÓN DE PARES SEMEJANTES}

El cambio urbano es un fenómeno complejo y dilatado en el tiempo, de manera que solamente es posible evidenciar cambios sustantivos en las ciudades y los barrios a medio y largo plazo. De hecho, el análisis comparativo pone de manifiesto que lo más adecuado es hablar de 'reproducción urbana', pues las diferencias existentes entre barrios se mantienen a lo largo del tiempo (Brama, A., 2013; Navarro, C., 2013; Navarro, C. et al., 2015; Wei, F. et al., 2014). En buena medida, el propósito de los procesos de regeneración urbana es cambiar esa pauta: al comparar un momento anterior y posterior a su desarrollo debería observarse un mayor grado de cohesión entre diferentes territorios (entre ciudades y/o barrios en una ciudad). Aun así, los escasos análisis evaluativos sobre estos procesos ponen de manifiesto que los impactos son bastante modestos (Lawless, P., 2007; O’Reilly, D., 2007; Thomson, H., 2008).

Pese a ello, uno de los grandes retos en el estudio de las políticas de regeneración urbana se encuentra en la evaluación de sus impactos. Esto se debe, fundamentalmente, a tres cuestiones. Una de carácter 'informativo', pues, normalmente, la evaluación suele centrarse más en aspectos relativos a la gestión o el alcance de resultados (niveles de ejecución, eficacia o eficiencia) que a la medición de sus impactos sobre la comunidad donde se desarrollan. Otra de carácter sustantivo, porque suelen evaluarse atendiendo únicamente a los resultados alcanzados, sin plantear de una forma clara cuál es la 'teoría' que subyace al programa y/o proyectos, que debiera explicar cuáles son los impactos previstos y por qué. Y, por último, otra de orden metodológico, en atención a las estrategias usadas con mayor frecuencia para evidenciarla existencia de impactos de los proyectos (Rhodes, J. et al., 2005).

Esto último se deriva de los diseños más comúnmente empleados para estudiar los proyectos de regeneración urbana; a saber: la consulta a informantes/expertos y los estudios de caso. Como es sabido el primer acercamiento ofrece información cualificada sobre el desarrollo e impactos de los programas de regeneración urbana a partir de la visión que tienen las personas consultadas, siendo sensible al proceso de selección de los informantes y/o dependiente de su posición y percepción (Taylor, D. y Balloch, S., 2005). Por su parte, los estudios de casos, que suelen centrarse en presentar casos ejemplares ('buenas prácticas'), o al contrario, en casos que muestran los efectos negativos de la regeneración urbana (principalmente, respecto al fenómeno de la gentrificación ${ }^{3}$ ), pero sin

\footnotetext{
${ }^{3}$ Gentrificación alude al proceso mediante el cual la población original de un barrio, generalmente céntrico y popular, es progresivamente desplazada por otra de un nivel adquisitivo mayor. Este término, acuñado en el contexto urbano londinense (Glass, R., 1964), hace referencia a procesos de renovación urbana de viejas zonas céntricas de las ciudades protagonizado por individuos de clase media que rehabilitan la deteriorada edificación residencial, haciendo subir los precios de la vivienda y provocando la expulsión de las clases obreras que originalmente habían ocupado el sector (Díaz, I., 2013). Por lo tanto, la gentrificación implica reestructuración urbana y recambio de clases sociales (Clark, E., 2005). Revista de Estudios Andaluces, núm. 36 (2018) pp. 147-177. e-ISSN: 2340-2776
} http://dx.doi.org/10.12795/rea.2018.i36.07

(c) (i) $\ominus$

Esta obra se distribuye con la licencia Creative Commons Reconocimiento-NoComercial-SinObraDerivada 4.0 Internacional (CC BY-NC-ND 4.0.) 
ofrecer una visión comparativa que permita generalizar sus resultados. En todo caso, este acercamiento metodológico puede resultar idóneo para comprobar si una explicación previamente formulada y/o analizada se produce también -o no- en otros contextos donde no se ha aplicado con anterioridad, o bien para indagar en profundidad procesos que pueden dar lugar a la formulación de explicaciones que pueden analizarse y comprobarse en otros casos (Hamel, J., 1993). En estas dos estrategias una posible mejora es aumentar el número de casos/informantes para garantizar que existe cierto volumen y variedad entre ellos que permita realizar ciertas comparaciones (Smelser, N.J., 1982). Ahora bien, estas dos estrategias enfrentan una limitación común: el hecho de no poder atribuir de forma convincente impactos a los proyectos de regeneración urbana porque no existen evidencias de que el cambio habido antes y después de la intervención se deba a otras razones que pueden darse en áreas urbanas donde no se ha desarrollado un proyecto de regeneración (Rossi, P.H., 1999).

Como punto de partida los programas y proyectos de regeneración urbana tienen como objeto mejorar la calidad de vida en las áreas territoriales en las que actúan (o, al menos, algunos de sus aspectos). Esto supone que la evaluación de su impacto pasa por comparar si la calidad de vida, o mejor, aquellos aspectos que son objeto de sus actuaciones, presentan cambios en momentos anteriores y posteriores a la ejecución del proyecto (o intervención). Este es el ejercicio que suele desarrollarse cuando se recurre a la consulta a expertos o se analiza la evolución de barrios concretos en donde se han aplicado proyectos (sea mediante estudios de casos en profundidad, sea mediante un diseño cross-sectional). Ahora bien, esta comparación temporal puede ser insuficiente porque el cambio observado puede deberse a otros factores diferentes al proceso de intervención, acontecimientos o políticas públicas que también se dan en otras áreas urbanas. Una de las formas más adecuadas de asegurar en mayor medida que el cambio se debe al proyecto se encuentra en el establecimiento de contrafactuals, a saber: evidencias sobre lo que ocurre en otras áreas similares donde no se han desarrollado proyectos. O de manera más coloquial, planteándose la siguiente pregunta: ¿qué hubiese ocurrido si no se hubiese desarrollado el programa de regeneración urbana?

Existen diferentes formas para establecer comparaciones que permitan responder a esa pregunta. En el caso que nos ocupa una de las estrategias más oportunas consistiría en 'la selección de pares semejantes', esto es, elegir una zona que sea la más similar posible a aquella donde se ha desarrollado el proyecto en un momento previo al inicio de este último; esto es, un 'área experimental' y otra que sea 'equivalente' a esa. Si se prefiere, que las dos zonas tengan las 'mismas condiciones de partida' antes de la aplicación del proyecto de regeneración urbana. De este modo, si al comparar el cambio habido entre ese momento anterior a la aplicación del programa y un momento posterior existen diferencias entre el área donde se ha intervenido y el área urbana similar donde no se ha intervenido, cabría atribuir esa diferencia con mayor seguridad al hecho de haber desarrollado el proyecto en las primeras. Por tanto, mediante la 'selección de pares semejantes' compararíamos lo que ocurre en un barrio donde se ha intervenido con lo que ocurre en un barrio similar donde no se ha intervenido, mostrando este último evidencias sobre 'lo que hubiese ocurrido de no haberse desarrollado el proyecto'. La diferencia entre los pares semejantes sería, pues, una evidencia del impacto del proyecto.

Revista de Estudios Andaluces, núm. 36 (2018) pp.147-177. e-ISSN: 2340-2776 http://dx.doi.org/10.12795/rea.2018.i36.07

(c) $($ ) $\odot \ominus$

cc) Esta obra se distribuye con la licencia Creative Commons Reconocimiento-NoComercial-SinObraDerivada 4.0 Internacional (CC BY-NC-ND 4.0.) 
Evidentemente, al igual que en otros diseños, la atribución de impacto al proyecto a partir del análisis de la diferencia entre los pares semejantes mejora si se aumenta el número de casos que se analizan, en la medida en que se tienen más evidencias comparativas (contractuals). Pero también es más relevante, o crítico, el modo en el que se seleccionan los pares para garantizar que las condiciones de partida entre ellos sean semejantes, pues de ello depende que la diferencia en el cambio temporal pueda atribuirse al desarrollo del proyecto. Por tanto, debemos dar respuestas a, al menos, dos cuestiones: ¿cuántos pares semejantes se analizan?, ¿respecto a qué son semejantes?

En relación a la primera cuestión, en general la respuesta es 'cuanto más, mejor' porque ampliar el número de casos permitirá realizar más comparaciones y tener en cuenta más características de los casos a la hora de hacer los análisis, pudiendo hacer más creíble la atribución del impacto de los proyectos. En nuestro caso la limitación es el número de proyectos que analizamos, un total de 81, un número de casos que garantiza, en principio, bastante credibilidad en la atribución de efectos a los proyectos, en lo que se refiere a los análisis que pueden desarrollarse. Respecto a la segunda cuestión debe establecerse cuáles son las condiciones de partida que son relevantes respecto a los proyectos, o mejor, aspectos sobre los que se pretende tener un efecto y/o aspectos que, según la 'teoría' del proyecto, afectan a los posibles impactos. Por tanto, debe diseñarse una estrategia de la selección de pares semejantes que atienda a tales condiciones de partida, así como evaluar si esa estrategia garantiza la posibilidad de realizar comparaciones entre ellos porque son similares en un momento anterior al inicio de los proyectos.

Así pues, desde un punto de vista operativo, ¿qué entendemos por áreas experimentales y equivalentes? En lo que sigue, un 'área experimental' es un área territorial de carácter urbano en la que tiene lugar la aplicación de un proyecto de actuación de alguno de los tres programas que se analizan, esto es, ARB, URBAN o ZNTS. En este último caso el área viene a coincidir con uno de los barrios existentes en la ciudad, parte o una agrupación de ellos, según la delimitación que ofrece el Instituto de Estadística y Cartografía de Andalucía (IECA). En los otros casos, el área territorial viene fijada por el proyecto, pudiendo ser tanto un conjunto de secciones censales, un barrio o un conjunto de barrios limítrofes.

Un 'área equivalente' sería un área geográfica de carácter urbano en la que no se desarrolla ningún proyecto de los programas de regeneración urbana que se analizan y tiene características físicas y socioeconómicas similares a un área experimental de la misma ciudad en un momento previo al inicio del proyecto que se desarrolla en esta última; pudiendo ser, pues, tanto una agrupación de secciones censales adyacentes, un barrio o una agrupación de los mismos. Se trata, pues, de áreas urbanas similares a las experimentales porque presentan las mismas 'condiciones de partida', de manera que, de haber cambios entre ellas entre un momento anterior y posterior al desarrollo del proyecto, cabría atribuir tal diferencia al proyecto desarrollado.

Por otro lado, en razón a los proyectos que se desarrollan, los criterios de selección para las áreas territoriales (barrios) que serán objeto de intervención deben dar cuenta de espacios urbanos que enfrentan retos de orden físico, social, económico y ambiental. Se trata de barrios que presentan cierto grado de vulnerabilidad urbana, al menos, en comparación con otras zonas de la misma ciudad. Por tanto, las condiciones de partida de las áreas experimentales y las áreas equivalentes 
deben referirse, al menos, al hecho de presentar los mismos niveles de vulnerabilidad urbana, que abarca tanto aspectos físicos, como socioeconómicos. Pero también es cierto que las reglas de selección y los objetivos más específicos de cada programa y sus proyectos hacen que en el conjunto de 'áreas experimentales' exista bastante heterogeneidad.

Esto se debe, aunque no únicamente, a dos lógicas (intra-urbana e inter-urbana) que dan cuenta de diferentes tipos de contextos de intervención: cascos históricos de ciudades medias, cascos históricos de grandes ciudades y barriadas. Esto supone que debe prestarse atención a estas dos lógicas al elegir las áreas equivalentes. Pero también porque otros ejercicios evaluativos han mostrado que el contexto local (la ciudad), tanto por sus dinámicas socioeconómicas, como también por sus dinámicas sociopolíticas, pueden intervenir en el impacto de los proyectos de regeneración urbana; en especial en programas que se caracterizan por estar sometidos a lógicas de gobernanza multinivel, como los que analizamos aquí (Hollister et al., 1995). Esto es, puede existir un 'efecto ciudad'. Por ello, como punto de partida, las áreas equivalentes deben tener un nivel similar de vulnerabilidad urbana que las áreas experimentales y situarse en la misma ciudad, en el mismo municipio, pero no ser vecinas de áreas territoriales experimentales para así evitar un posible efecto de difusión.

\section{METODOLOGÍA Y CRITERIOS PARA LA DELIMITACIÓN DE ÁREAS EQUIVALENTES}

Para delimitar las áreas territoriales de los proyectos se ha partido de la información proporcionada por las instituciones que desarrollan los tres programas, ajustándola a los límites de las secciones censales para el año 2001, y posteriormente, a la delimitación de barrios que ofrece el IECA. En el caso del programa ARB, la Consejería de Fomento y Vivienda ha facilitado la delimitación territorial completa de los proyectos. En la mayoría de los casos de los proyectos URBAN la delimitación territorial viene recogida en la documentación del proyecto, ajustando mediante superposición, la información proporcionada con la delimitación de secciones censales y los barrios. En el caso del programa ZNTS no existe una delimitación explícita de los proyectos, como tampoco del ámbito territorial de las Unidades de Trabajo Social encargadas de desarrollarlos; $y$, de hecho, es frecuente encontrar situaciones de desarticulación territorial en los servicios sociales especializados (Cordero, G., 2000). Por ello, previa consulta a los responsables del programa en la Consejería para la Igualdad y Bienestar Social, se ha utilizado como delimitación de estos programas el barrio que en cada ciudad tienen la misma denominación que el proyecto.

La selección del área equivalente siempre será un barrio o agrupación de barrios dentro de la misma ciudad que el área experimental. Se ha escogido el nivel territorial del barrio que aporta el IECA porque constituyen, por lo general, áreas territoriales bastante homogéneas internamente y que además tienen una dimensión demográfica y de extensión adecuada, en principio, para constituirse como áreas territoriales en las que desarrollar procesos de regeneración urbana. Aunque, como veremos más adelante, en general las áreas territoriales de los proyectos suelen ser bastante más grandes, agrupando a varios barrios 4 .

\footnotetext{
${ }^{4}$ Esto es especialmente destacable en el caso de la ciudad de Málaga, en la que los barrios que ofrece el IECA son pequeños y, en algunos casos, en exceso, llegando a delimitarse barrios con dimensiones similares a una sección censal. Revista de Estudios Andaluces, núm. 36 (2018) pp.147-177. e-ISSN: 2340-2776
} http://dx.doi.org/10.12795/rea.2018.i36.07

(c) (†) $\ominus$

cc) Esta obra se distribuye con la licencia Creative Commons Reconocimiento-NoComercial-SinObraDerivada 4.0 Internacional (CC BY-NC-ND 4.0.) 
Una vez delimitadas las áreas de los proyectos, esto es, las áreas experimentales, para elegir las áreas equivalentes se han utilizado los siguientes criterios (tabla 1), que se detallan a continuación:

Tabla 1. Criterios para la identificación de áreas equivalentes.

\begin{tabular}{|c|c|}
\hline Tipo de criterio & Criterio \\
\hline \multirow[t]{5}{*}{ Cuantitativos } & 1. Actuación \\
\hline & 2. $\quad$ Similitud \\
\hline & 3. Demográfico \\
\hline & 4. Situación I (centro-periferia) \\
\hline & 5. Situación II (distancia) \\
\hline Cualitativos & 6. Morfología urbana \\
\hline
\end{tabular}

Fuente: Elaboración propia.

Criterio 1: Ausencia de actuación de programas de regeneración urbana.

El área equivalente no deberá haber sido objeto de ninguna intervención por parte de los programas planteados que se analizan en el estudio, esto es: ARB, URBAN y ZNTS.

Criterio 2: Similitud socio-económica.

El área equivalente será lo más similar posible al área de actuación según sus características socioeconómicas ¿Cómo establecemos la similitud entre áreas experimentales y equivalentes?

10. Atendiendo al Indicador de Desigualdad Urbana Municipal (IDUM) ${ }^{5}$ con resultados para 2001 recogidos en el "Atlas de la Vulnerabilidad Urbana en España" realizado por el Ministerio de Fomento. Este indicador se ha seleccionado por relacionar una serie de variables a nivel de sección censal (población en paro, sin estudios, y viviendas sin servicio o aseo) respecto a la media del municipio. Con ello se obtiene el grado de vulnerabilidad de cada sección en el marco de la ciudad en la que sitúan.

Dado que las áreas territoriales de los proyectos suponen agregaciones de secciones censales se ha calculado la medida del IDUM de estas para medir el grado de vulnerabilidad del área en su conjunto.

2‥ Una vez obtenidos los valores del IDUM para las áreas experimentales, la distribución se ha clasificado en quintiles. De tal manera, que las posibles áreas equivalentes han de pertenecer al mismo quintil que el área experimental.

\footnotetext{
${ }^{5}$ EI IDUM se calcula sumando tres indicadores de vulnerabilidad "Porcentaje de población en paro", "Porcentaje de población sin estudios" y "Porcentaje de población en viviendas sin servicio o aseo", una vez estandarizados con respecto a los valores de su respectivo municipio por el método chi cuadrado, y aplicada una transformación logarítmica para ajustar la distribución.

Revista de Estudios Andaluces, núm. 36 (2018) pp. 147-177. e-ISSN: 2340-2776
}

http://dx.doi.org/10.12795/rea.2018.i36.07 


\section{Criterio 3: Demográfico.}

El área equivalente seleccionada además de que su valor de IDUM pertenezca al mismo quintil que el valor que toma el área experimental, tendrá que tener un volumen demográfico dentro de un margen del $\pm 50 \%$ del número de habitantes del área experimental. En caso de no encontrarse ningún área equivalente que cumpla este criterio, se amplía el margen de población hasta el $\pm 66 \%$ de la población. Con ello se pretende que el volumen de población que es objetivo de un proyecto (área experimental) sea similar al de su área equivalente.

Criterio 4: Situación o localización espacial en la ciudad: centro-periferia.

Este criterio se define por las relaciones con el entorno próximo y lejano; depende por tanto de la posición de cada área territorial con respecto a otros lugares similares o complementarios y, en consecuencia, de la posición que le aseguran, que viene definida por las relaciones con estos. En nuestro caso, se han considerado dos posibles localizaciones, a saber: de central o de periferia. Y a partir de ahí, dos tipos de áreas territoriales: por un lado, las que pertenecen al centro histórico de la ciudad, y por otro, las que no pertenecen al centro histórico, y a las que hemos denominado de manera genérica como periféricas.

Teniendo en cuenta esta aclaración, el área equivalente deberá pertenecer a la misma tipología de barrio que el área experimental en cuanto a su localización. Así, un área experimental que pertenezca al centro histórico de una ciudad únicamente podrá ser comparable, y por tanto tener su equivalente, en otra área del centro histórico, e igual ocurrirá con las áreas experimentales que tienen su área de actuación fuera del centro histórico, siendo solamente comparables con otra área periférica.

Pese a lo establecido con anterioridad, este criterio requiere modificar la interpretación respecto al 'criterio de actuación'. Dado que existen proyectos que actúan en todo el centro histórico, siguiendo estrictamente el criterio de actuación cualquier área del centro histórico quedaría excluida como posible equivalente. No obstante, en estos casos creemos necesario establecer una excepción, pues consideramos que un área del centro histórico solamente es comparable en sus características, funciones y procesos urbanos y sociales con otra área del centro histórico. En estos casos se delimita dentro del centro histórico un área experimental en el barrio o barrios donde se hayan concentrado las actuaciones de los proyectos, dejando como área equivalente el barrio o barrios donde se ha actuado menos.

Criterio 5: Localización relacional: distancia o vecindad.

Este criterio se refiere a la importancia que desempeña la distancia. Por una parte, la distancia puede aumentar o frenar las interacciones entre áreas territoriales, y por otra permite establecer un indicador en función de su situación geográfica relativa.

Revista de Estudios Andaluces, núm. 36 (2018) pp.147-177. e-ISSN: 2340-2776 http://dx.doi.org/10.12795/rea.2018.i36.07

(c) $($ ) $(\ominus)$

cc) Esta obra se distribuye con la licencia Creative Commons Reconocimiento-NoComercial-SinObraDerivada 4.0 Internacional (CC BY-NC-ND 4.0.) 
Teniendo en cuenta el enunciado de Tobler: "todo está relacionado con todo, pero las cosas próximas están más relacionadas que las distantes" (Tobler, W., 1979), con este criterio se pretende precisamente evitar la selección como área equivalente de áreas que sean vecinas de la experimental, pues pretendemos evitar los posibles procesos de difusión espacial, entendidos como la acción, y el resultado de la acción, de expandirse, o de transmitir y propagar de manera uniforme los efectos de la actuación practicada en las áreas experimentales. Es decir, queremos comparar dos barrios similares pero no nos interesa que sean vecinos, pues entre barrios vecinos existirá mayor posibilidad de producirse procesos de difusión.

En suma, para que un área sea considerada como equivalente, esta área no podrá ser vecina al área experimental en que se ha actuado por parte de cualquiera de los programas de regeneración urbana considerados, evitando con ello la posibilidad de fenómenos de difusión espacial.

Más concretamente, se entiende que dos áreas no son vecinas cuando no comparten lindes en común. Para ello se ha realizado un análisis espacial de proximidad en la que se contemplan como posibles áreas equivalentes el barrio o barrios situados a una distancia mayor de cero metros del área experimental. De esta manera, al quedar representados los barrios en forma de polígonos, el área equivalente deberá tener al menos un barrio intermedio (un polígono) de distancia entre el área experimental y el barrio equivalente. Es lo que se puede llamar un "barrio en transición" que evitará, o al menos reducirá, la influencia, la difusión espacial del programa de regeneración del área experimental sobre el área equivalente. Tras operar con el análisis espacial de proximidad, se otorgó el valor 1 a los barrios que presentan vecindad, es decir, si existe una frontera común entre las regiones analizadas, y valor cero, si no existe ningún tramo fronterizo común. Así pues, estamos usando un criterio de lejanía, pues entendemos que al estar más alejado el posible barrio equivalente se pierde la influencia de los fenómenos de difusión.

Como veremos en el apartado de resultados, no siempre podremos seleccionar como equivalentes áreas lejanas a las experimentales, de hecho, en ocasiones áreas experimentales y equivalentes son vecinas desde el punto de vista geométrico. En estos casos, se ha tenido en cuenta la existencia de barreras físicas (avenida, río, vías del ferrocarril, amplias zonas sin urbanizar, etc.), para considerar que dos barrios con lindes en común, no son vecinos al existir una barrera física que impide, o cuando menos hace que pierda fuerza, el fenómeno de la difusión.

\section{Criterio 6: Morfología urbana.}

Por último, a los criterios anunciados previamente, de corte cuantitativo, se ha incorporado un criterio cualitativo basado en el conocimiento propio y en la observación a través de ortofoto ${ }^{6}$ de los barrios de las ciudades en base a sus características, y operada en Sistemas de Información Geográfica (SIG) a través de la superposición de la coberturas para las áreas experimentales y actuaciones. Así, para la identificación de las áreas equivalentes se ha considerado el aspecto formal de los barrios, teniendo en cuenta su emplazamiento, situación urbana, poblamiento (disperso o concentrado), el plano (irregular, ortogonal, lineal, radio-céntrico), la construcción (edificación,

\footnotetext{
${ }^{6}$ Instituto de Estadística y Cartografía de Andalucía: Ortofotografía Pancromática 0,5 metros/pixel.2001.

Revista de Estudios Andaluces, núm. 36 (2018) pp. 147-177. e-ISSN: 2340-2776

http://dx.doi.org/10.12795/rea.2018.i36.07
} 
colectiva o individual; densificación, alta o baja; trama urbana, abierta o compacta), y usos del suelo (residencial, comercial, industrial, equipamientos).

Con todo lo planteado, y a modo de resumen, se puede decir que la expresión para la obtención del área equivalente sería la siguiente, que se presenta también esquemáticamente en la tabla 2.

Equivalente $=A$. sin actuación + Similitud (desigualdad IDUM=quintil) + Población $( \pm 50-66 \%)+$ Situación I (Centro Vs Centro / Periferia Vs Periferia) + Situación II (distancia >0 metros) + Morfología urbana.

Tabla 2. Criterios para la identificación de áreas equivalentes: delimitación y aplicación.

\begin{tabular}{|c|c|c|c|}
\hline Tipo de criterio & Criterio & Delimitación & Área equivalente \\
\hline \multirow{5}{*}{$\begin{array}{c}\text { Criterios } \\
\text { cuantitativos }\end{array}$} & Actuación & $\begin{array}{l}\text { Área que no ha sido objeto } \\
\text { de intervención de } \\
\text { programas ARB, URBAN o } \\
\text { ZNTS }\end{array}$ & $\begin{array}{l}\text { Área sin actuación (ARB, } \\
\text { URBAN, ZNTS) }\end{array}$ \\
\hline & Similitud & $\begin{array}{ll}\text { Mismo nivel } & \text { de } \\
\text { vulnerabilidad urbana } & \end{array}$ & $\begin{array}{l}\text { Mismo quintil del valor IDUM } \\
\text { del A. Experimental }\end{array}$ \\
\hline & Demográfico & $\begin{array}{l}\text { Población similar a la del } \\
\text { área experimental }\end{array}$ & $\begin{array}{l}\text { Población } \pm 50-66 \% \text { del A. } \\
\text { Experimental }\end{array}$ \\
\hline & $\begin{array}{l}\text { Situación I } \\
\text { (centro-periferia) }\end{array}$ & $\begin{array}{l}\text { Misma localización en la } \\
\text { ciudad (centro o periferia) }\end{array}$ & $\begin{array}{l}\text { B. Experimental = centro } \\
\text { histórico / B. Equivalente = } \\
\text { centro histórico } \\
\text { B. Experimental = periferia / B. } \\
\text { Equivalente = periferia }\end{array}$ \\
\hline & $\begin{array}{l}\text { Situación II } \\
\text { (distancia) }\end{array}$ & & $\begin{array}{l}\text { distancia mayor cero } \rightarrow \\
\text { existencia de un 'barrio de } \\
\text { transición'; y/o barreras } \\
\text { urbanas importantes }\end{array}$ \\
\hline $\begin{array}{c}\text { Criterios } \\
\text { cualitativos }\end{array}$ & Morfología urbana & $\begin{array}{l}\text { Similar morfología urbana } \\
\text { (tipología edificios, espacios) }\end{array}$ & \\
\hline
\end{tabular}

Fuente: Elaboración propia.

\section{RESULTADOS: ÁREAS EXPERIMENTALES Y EQUIVALENTES OBTENIDAS}

En la mayoría de los casos, la aplicación de los criterios ha dado como resultado la posibilidad de elegir entre distintas áreas equivalentes en cada ciudad. En este caso, se ha seleccionado aquella que en mayor medida se ajustaba a los criterios delimitados. Ahora bien, en algunos casos (proyectos) se han tenido que aplicar criterios ad-hoc. Estos criterios han sido, principalmente, de cuatro tipos, y su aplicación ha sido la siguiente:

1. Tamaño demográfico del área experimental: aunque aparecen áreas equivalentes mediante los otros criterios, no se puede delimitar un área equivalente debido a la gran la extensión y tamaño demográfico del área experimental. Para este caso se han dado dos soluciones o criterios ad-hoc: 
1.1. Reducción del área experimental a uno de los barrios y/o secciones incluidos en atención a la intensidad de la intervención realizada, buscando así un área equivalente similar en cuanto a extensión y tamaño demográfico. Este ha sido el caso de:

- Proyecto ARB en 'Casco Norte' (Sevilla). El área de este programa queda delimitada por el conjunto de los barrios de Encarnación-Regina, Santa Catalina, San Vicente, Feria, San Julián, San Gil, San Lorenzo. Se trata de una extensión y volumen demográfico muy grandes. Tras cerciorarnos que muchas de las actuaciones se concentraban en el barrio de Feria (además de ser el origen de la actuación en la zona), decidimos incluir en el proyecto únicamente dicho barrio. De esta manera, y al reducir la población, obtenemos como área equivalente el barrio de San Bartolomé; que cumple con los criterios establecidos, a saber: no haber sido objeto de intervención, similitud socio-económica según IDUM; demográfica, y que presenta las características, en cuanto a morfología urbana, de lo que hemos denominado 'barrio céntrico' (en contraposición a 'barrio periférico').

- Proyectos ARB y ZNTS en 'Piñera-Saladillo' (Algeciras). En los proyectos originales, tanto de ARB como ZNTS, en el área estaban incluidos los barrios de la Piñera y de Saladillo. Tras comprobar que la gran mayoría de las actuaciones se localizaban en el barrio de la Piñera se decidió reducir el área experimental a dicho barrio. Obteniendo de esta manera como equivalencia para ambos proyectos el barrio de San Bernabé Oeste.

1.2. Agregación de áreas equivalentes: se trata del caso en el que aparecen áreas equivalentes pero sin cumplir el criterio demográfico establecido. En este caso se han agrupado barrios vecinos que cumplían con los otros criterios. Algunos de los equivalentes obtenidos en el primer listado no eran similares a los experimentales, en cuanto a su morfología urbana: tipo de vivienda, estado del edifico, entramado de las calles, etc.

- Proyectos ARB 'El Puche' y ARB 'Chanca Pescadería' (Almería). En un primer momento, se obtuvieron como equivalentes para ambas 'áreas' los barrios de 'Centro Rambla' y 'Nueva Almería'. Estos dos últimos poseen en la actualidad un hábitat urbano que difiere de las características de barrios tan singulares como El Puche o la Chanca, por lo que se optó por otras áreas equivalentes. En concreto se agruparon barrios potencialmente equivalentes y que por sí solos no aparecían como tal debido al criterio de población, formando nuevas áreas como conjunto de barrios. Así, el área equivalente de los ARB 'Puche' y 'ChancaPescadería' es la conjunción de los barrios Los Almendros, Piedras Redondas y Araceli (con un total de 4.822 habitantes).

- Proyecto ARB 'Ajerquía' (Córdoba). Conformado por los barrios de San Lorenzo, Santa Marina y San Andrés-San Pablo se propone como equivalente la agrupación de los barrios de La Magdalena, Cerro de la Golondrina y Ribera. Entre los tres barrios suman un total de 9.356 habitantes, ajustándose de esta manera al criterio poblacional (+ - 50\%), ya que el área de Ajerquía está bastante poblada (12.037 habitantes), además de respetar los otros criterios utilizados para definir áreas equivalentes.

- Proyecto ZNTS 'Distrito 6, Pérez Cubillas' (Huelva). Con los criterios establecidos a priori, se obtenían unos equivalentes que nadan tenían que ver con el área experimental. Se decidió por ello agrupar dos barrios potencialmente equivalentes pero que por sí solos no lo podían 
ser debido a que no cumplían el criterio de tamaño poblacional de los siguientes barrios. La solución fue agruparlos: Barriada Nuestra Señora del Rocío, Barriada de la Cinta (2.186 habitantes).

- Proyecto ZNTS 'Tres Barrios' (Sevilla). En un primer listado se obtuvo como equivalente La Plata, pero este barrio ya se había decidido que fuera la equivalencia de otras áreas experimentales; ARB/URBAN/ZNTS de Polígono Sur. Por este motivo y para evitar más superposiciones, se optó por los siguientes barrios: San Jerónimo, La Bachillera (total de 11.433 habitantes).

2. Amplitud de la cobertura territorial de las áreas experimentales: se trata, fundamentalmente, de centros históricos que son, en su totalidad, delimitados como área en la que se interviene. En estos casos, y para salvaguardar la equivalencia en atención a la localización en la ciudad y su morfología, se ha optado por definir áreas experimentales para cada programa atendiendo al barrio en el que en mayor medida se produce la actuación, eligiendo áreas equivalentes en el propio centro histórico. Esto supone no atender al criterio de 'no vecindad', pero garantiza la existencia de áreas equivalentes con similares rasgos urbanos, demográficos y socioeconómicos. Así pues, el criterio ad-hoc utilizado ha sido dividir el área experimental escogiendo una parte como experimental (donde hay más intervención) y otra como equivalente. Este ha sido el caso de:

- Proyecto ARB 'Centro Histórico' (Jerez): se propone tener en cuenta solamente el barrio de 'Santiago' como experimental, obteniendo de esta manera como equivalente el barrio de Picadueñas.

- URBAN 'Zona Sur de Jerez'; se propone delimitar únicamente como experimental el barrio de 'San Telmo Viejo' y el de Santo Tomás de Aquino como equivalente.

- Proyecto ARB 'Bajo Albaicín' (Granada). Como el propio nombre del proyecto indica, la intervención no se reparte de forma equilibrada en todo el barrio del Albaicín, sino que existen mayor número de actuaciones en la mitad sur del barrio. Optamos por reducir el área experimental a las secciones que se corresponden con la mitad sur del barrio y la equivalente en la mitad norte del barrio. Aunque no se cumple el criterio de vecindad, se cumplen todos los demás.

3. Superposición entre proyectos de diferentes programas: en algunos casos existe la superposición entre dos y tres programas, por lo que distintos proyectos se ejecutan en una misma área territorial. Así, aunque como proyectos son diferentes, se trata de la misma área territorial, y, por tanto, se ha definido una misma área equivalente. Esto hace que, en su conjunto, aunque existan tantas áreas experimentales como equivalentes, el número de proyectos sea superior. Es el caso de:

- 'Polígono Sur' (Sevilla), presente en ARB, URBAN Y ZNTS. En los tres proyectos el área de actuación queda definida por los barrios de La Oliva, Avenida de La Paz, Letanías y el barrio 'Polígono Sur' propiamente dicho. Es lógico, por tanto, que el equivalente para las tres actuaciones sea el mismo: el barrio, que según los criterios establecidos, es el barrio de La Plata, que cuenta con una población residente de 13.537 habitantes.

Revista de Estudios Andaluces, núm. 36 (2018) pp.147-177. e-ISSN: 2340-2776 http://dx.doi.org/10.12795/rea.2018.i36.07 
- 'Centro Histórico de Cádiz': se desarrollan dos programas (ARB y URBAN), pero, además, en principio, su cobertura es para todo el casco antiguo. Aquí se ha aplicado tanto el criterio adhoc derivado de la superposición, como el anterior, referido a la cobertura del área experimental. Así, dentro del casco antiguo de la ciudad, se ha definido tanto un área experimental más restringida para cada proyecto, como áreas equivalentes, en atención a la localización de actuaciones. En el caso de Cádiz, el ARB primigenio 'Recinto histórico de Cádiz' queda dividido en dos, a saber: ARB 'Recinto histórico de Cádiz l' (compuesto por los céntricos barrios del 'Pópulo' y 'Santa María' y el ARB 'Recinto histórico de Cádiz II' ('San Juan', 'Callejones' y 'La Viña'). A su vez el URBAN queda de la siguiente manera: compuesto por los barrios de 'Mentidero', 'San Antonio' y 'Falla'. Para todas estas zonas experimentales se proponen dos áreas equivalentes. La primera compuesta por los barrios San Felipe, Hospital de mujeres y Candelaria. La segunda la conforman los barrios de San Francisco Mina y Alameda-San Carlos.

4. Misma área equivalente para diferentes áreas experimentales: en algunas ciudades a diferentes áreas experimentales le corresponde la misma equivalencia. Esto se debe a que de los equivalentes que se obtenían en la primera aproximación únicamente un área era similar, desde un punto de vista de morfología urbana, a las zonas experimentales definidas en los proyectos. Esto hace, y siguiendo lo que se ha dicho en el criterio ad-hoc no 3, que, aunque existan tantas áreas experimentales como equivalentes, el número de estas últimas sea inferior. Ese es el caso de:

- 'San Bernabé Oeste' de Algeciras (también incluido en el criterio ad-hoc 1.1) equivalente de las áreas ARB/ZNTS 'La Piñera'.

- 1을 San Felipe, Hospital de Mujeres, Candelaria; 20 San Francisco Mina y Alameda-San Carlos en Cádiz (también incluidos en el criterio ad-hoc 3): equivalente de: ARB 'Recinto histórico de Cádiz I', compuesto por los céntricos barrios del 'Pópulo' y 'Santa María'; ARB 'Recinto histórico de Cádiz II', formado por 'San Juan', 'Callejones' y 'La Viña'. Y del URBAN compuesto por los barrios de 'Mentidero', 'San Antonio' y 'Falla'.

- 'Los Almendros, Piedras Redondas y Araceli' en Almería (también incluidos en el criterio adhoc 1.2): equivalente de cuatro áreas experimentales: ARB/ZNTS de 'El Puche' y 'La ChancaPescadería'.

- Las 'Moreras' (Córdoba): equivalente de dos áreas experimentales: del ARB 'Barriada San Martín de Porres' y ZNTS 'Palmeras'.

- Polígono La Fuensanta (Córdoba): equivalente de dos áreas experimentales: URBAN 'Sur de Córdoba' y ZNTS 'Sector Sur'.

- 'Cerrillo de la Maracena' (Granada): equivalente de dos áreas experimentales: ARB 'Almanjáyar' y ZNTS 'D. Norte (Cartuja)'.

- 'Barriada del Carmen' (Huelva): equivalente de tres áreas experimentales: ARB 'Marismas del Odiel'; ZNTS 'Marismas del Odiel' y ZNTS ‘Distrito 5 (Torrejón)'.

- 'Peñafecit-Estación' (Jaén): equivalente de dos áreas experimentales: ARB 'Centro histórico de Jaén' y 'Plan URBAN III de Jaén'. 
En su conjunto, la aplicación de los criterios generales, junto los criterios ad-hoc, han dado lugar a la selección de las áreas equivalentes que se muestra en las tablas y mapas siguientes para cada una de las ciudades y proyectos de regeneración urbana.

Tabla 3. Áreas experimentales y equivalentes en Algeciras.

\begin{tabular}{|l|l|c|c|c|c|c|c|}
\hline \multirow{2}{*}{ Programa } & \multirow{2}{*}{ Proyecto } & \multicolumn{4}{|c|}{ Área experimental } & \multicolumn{3}{c|}{ Área equivalente } \\
\cline { 3 - 8 } & Denominación & $\begin{array}{c}\text { Población } \\
\text { (hab.) }\end{array}$ & $\begin{array}{c}\text { Valor IDUM } \\
\text { (quintil) }\end{array}$ & Denominación & $\begin{array}{c}\text { Población } \\
\text { (hab.) }\end{array}$ & $\begin{array}{c}\text { Valor IDUM } \\
\text { (quintil) }\end{array}$ \\
\hline ARB/ZNTS & $\begin{array}{c}\text { Piñera- } \\
\text { Saladillo }\end{array}$ & Piñera & 6.467 & $-0,19(3)$ & $\begin{array}{c}\text { S. Bernabé } \\
\text { Oeste }\end{array}$ & 5.556 & $-0,34(3)$ \\
\hline
\end{tabular}

Fuente: Elaboración propia.

Mapa 1. Áreas experimentales y equivalentes en Algeciras.

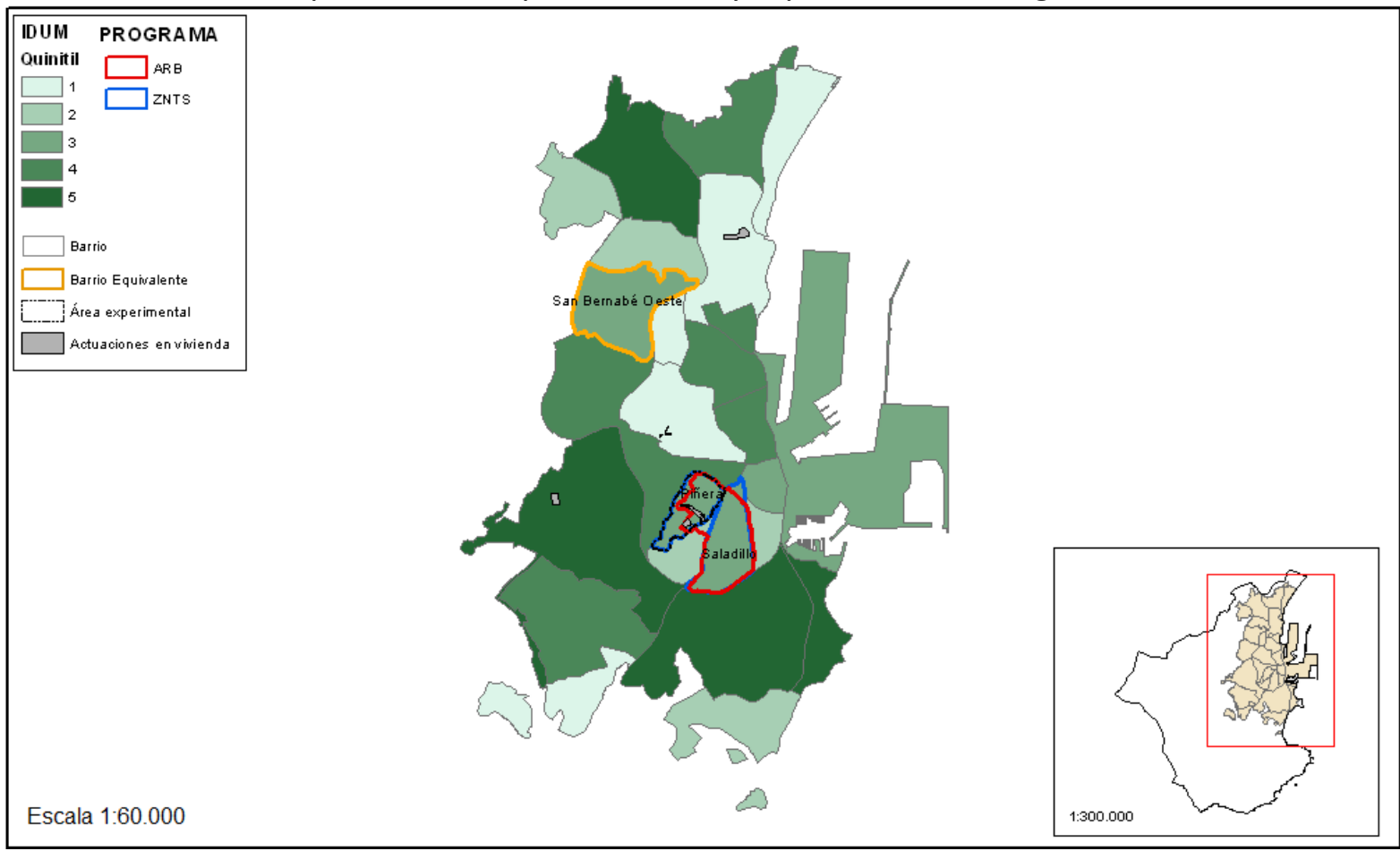

Fuente: Elaboración propia.

Revista de Estudios Andaluces, núm. 36 (2018) pp.147-177. e-ISSN: 2340-2776 http://dx.doi.org/10.12795/rea.2018.i36.07

\section{(c) (i) $\ominus$}


La Evaluación de las Políticas de Regeneración Urbana.

Propuesta Metodológica para la Delimitación de Áreas Experimentales y Equivalentes

Tabla 4. Áreas experimentales y equivalentes en Almería.

\begin{tabular}{|c|c|c|c|c|c|c|c|}
\hline \multirow[b]{2}{*}{ Programa } & \multirow[b]{2}{*}{ Proyecto } & \multicolumn{3}{|c|}{ Área experimental } & \multicolumn{3}{|c|}{ Área equivalente } \\
\hline & & Denominación & $\begin{array}{l}\text { Población } \\
\text { (hab.) }\end{array}$ & $\begin{array}{c}\text { Valor } \\
\text { IDUM } \\
\text { (quintil) }\end{array}$ & Denominación & $\begin{array}{l}\text { Población } \\
\text { (hab.) }\end{array}$ & $\begin{array}{c}\text { Valor } \\
\text { IDUM } \\
\text { (quintil) }\end{array}$ \\
\hline ARB/ZNTS & El Puche & El Puche & 4.328 & $5,55(5)$ & $\begin{array}{l}\text { Los Almendros, } \\
\text { Piedras Redondas } \\
\text { y Araceli }\end{array}$ & 4.822 & $2,07(5)$ \\
\hline ARB/ZNTS & $\begin{array}{l}\text { La Chancha- } \\
\text { Pescadería }\end{array}$ & $\begin{array}{l}\text { La Chancha- } \\
\text { Pescadería }\end{array}$ & 5.781 & $1,81(5)$ & $\begin{array}{l}\text { Los Almendros, } \\
\text { Piedras Redondas } \\
\text { y Araceli }\end{array}$ & 4.822 & 2,07 (5) \\
\hline URBAN & $\begin{array}{l}\text { Centro Hco. } \\
\text { de Almería }\end{array}$ & $\begin{array}{l}\text { Nicolás Salmerón, } \\
\text { Centro Paseo, Centro } \\
\text { Histórico, La Chanca }\end{array}$ & 16.992 & $0,83(5)$ & $\begin{array}{l}\text { Altamira, Oliveros } \\
\text { y Centro Rambla }\end{array}$ & 19.095 & $1,12(5)$ \\
\hline ZNTS & Fuentecica & $\begin{array}{l}\text { Fuentecica, } \\
\text { Quemadero }\end{array}$ & 4.355 & $0,20(2)$ & Barrio Alto & 6.845 & $-0,42(2)$ \\
\hline
\end{tabular}

Fuente: Elaboración propia.

Mapa 2. Áreas experimentales y equivalentes en Almería.

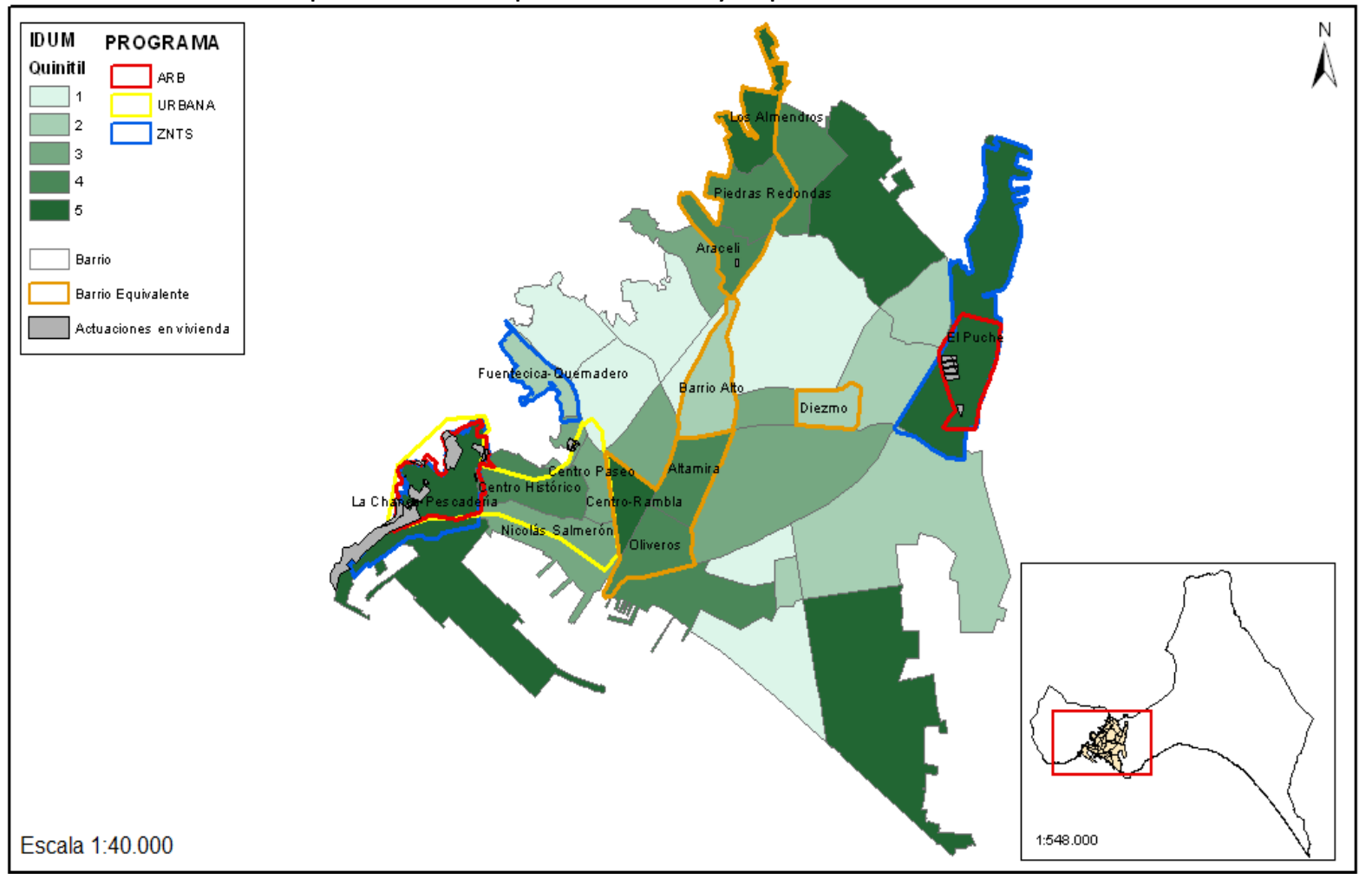

Fuente: Elaboración propia.

Revista de Estudios Andaluces, núm. 36 (2018) pp. 147-177. e-ISSN: 2340-2776

http://dx.doi.org/10.12795/rea.2018.i36.07

(c) (i) $\ominus$

Esta obra se distribuye con la licencia Creative Commons Reconocimiento-NoComercial-SinObraDerivada 4.0 Internacional (CC BY-NC-ND 4.0.) 
Tabla 5. Áreas experimentales y equivalentes en Cádiz.

\begin{tabular}{|c|c|c|c|c|c|c|c|}
\hline \multirow[b]{2}{*}{ Programa } & \multirow[b]{2}{*}{ Proyecto } & \multicolumn{3}{|c|}{ Área experimental } & \multicolumn{3}{|c|}{ Área equivalente } \\
\hline & & Denominación & $\begin{array}{c}\text { Población } \\
\text { (hab.) }\end{array}$ & $\begin{array}{c}\text { Valor } \\
\text { IDUM } \\
\text { (quintil) }\end{array}$ & Denominación & $\begin{array}{c}\text { Población } \\
\text { (hab.) }\end{array}$ & $\begin{array}{l}\text { Valor } \\
\text { IDUM } \\
\text { (quintil) }\end{array}$ \\
\hline ARB & $\begin{array}{l}\text { Recinto } \\
\text { Histórico } \\
\text { de Cádiz } 1\end{array}$ & $\begin{array}{c}\text { Pópulo y Sta. } \\
\text { María }\end{array}$ & 6.689 & $2,09(5)$ & $\begin{array}{l}\text { 1ㅇ. Selipe, Hospital de } \\
\text { Mujeres y Candelaria } \\
\text { 2o-S. Francisco Mina y } \\
\text { Alameda-S. Carlos }\end{array}$ & $\begin{array}{l}1 \text { 으 } 7.271 \\
\text { 2ㅇ } 7.145\end{array}$ & $-1,38(1)$ \\
\hline ARB & $\begin{array}{l}\text { Recinto } \\
\text { Histórico } \\
\text { de Cádiz } 2\end{array}$ & $\begin{array}{c}\text { S. Juan, } \\
\text { Callejones y la } \\
\text { Viña }\end{array}$ & 10.436 & $2,21(5)$ & $\begin{array}{l}\text { 1ㅇ. Selipe, Hospital de } \\
\text { Mujeres y Candelaria } \\
\text { 2o-S. Francisco Mina y } \\
\text { Alameda-S. Carlos }\end{array}$ & $\begin{array}{l}1 \text { ㅇ } 7.271 \\
2 \text { o } 7.145\end{array}$ & $-1,38(1)$ \\
\hline URBAN & $\begin{array}{l}\text { Urban de } \\
\text { Cádiz }\end{array}$ & $\begin{array}{l}\text { Mentidero, S. } \\
\text { Antonio y Falla }\end{array}$ & 6.582 & $-0,37(5)$ & $\begin{array}{l}\text { 10 S. Felipe, Hospital de } \\
\text { Mujeres y Candelaria, } \\
\text { 2oS. Francisco Mina y } \\
\text { Alameda-S. Carlos }\end{array}$ & $\begin{array}{l}1 \text { o } 7.271 \\
2 \text { ㅇ } 7.145\end{array}$ & $-1,38(1)$ \\
\hline ZNTS & La Paz & La Paz & 11.077 & $-0,15(2)$ & Loreto & 6.115 & $-0,57(2)$ \\
\hline
\end{tabular}

Fuente: Elaboración propia.

Mapa 3. Áreas experimentales y equivalentes en Cádiz.

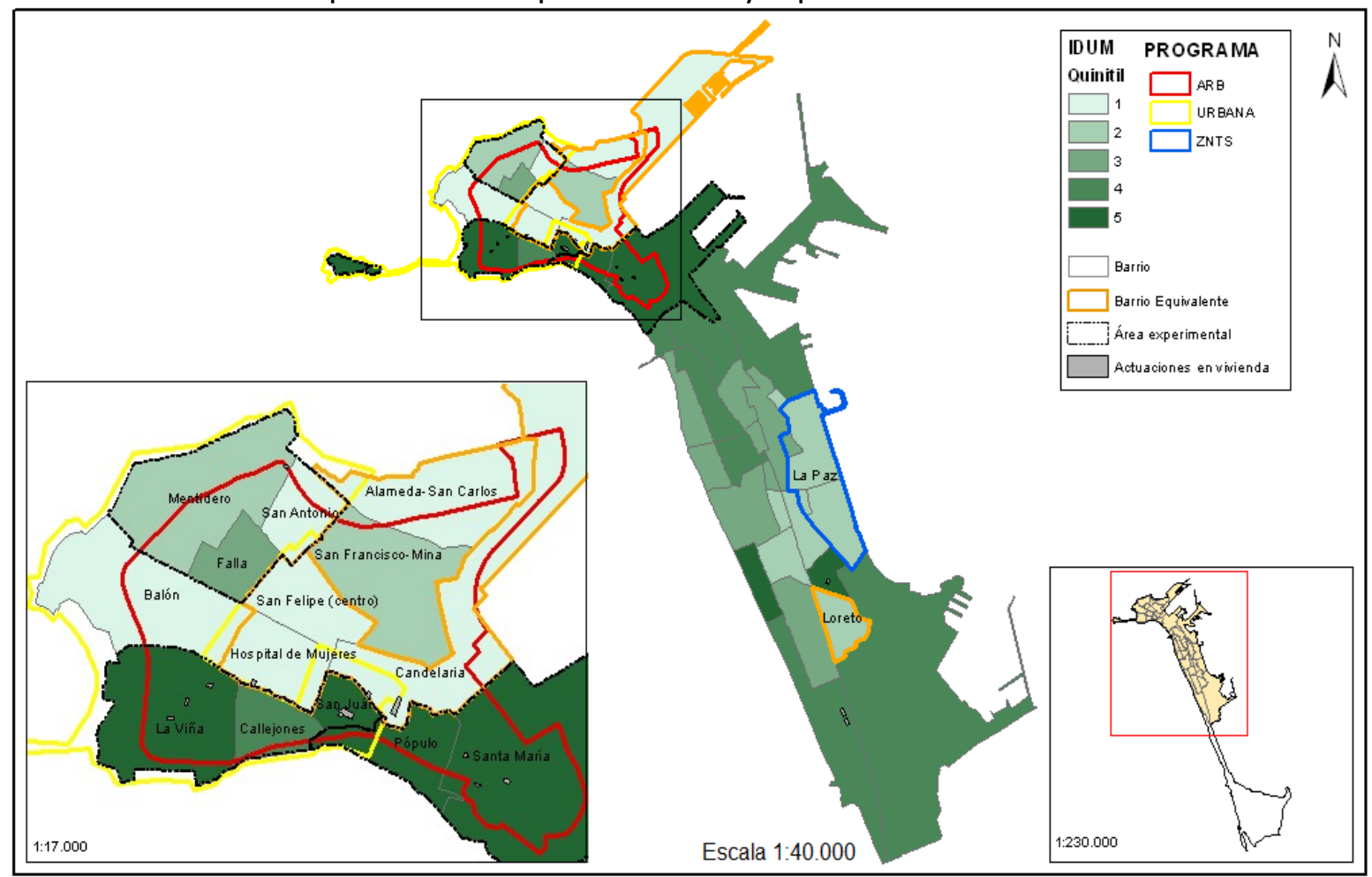

Fuente: Elaboración propia.

Revista de Estudios Andaluces, núm. 36 (2018) pp.147-177. e-ISSN: 2340-2776 http://dx.doi.org/10.12795/rea.2018.i36.07

\section{(c) (i) $\ominus$}


La Evaluación de las Políticas de Regeneración Urbana.

Propuesta Metodológica para la Delimitación de Áreas Experimentales y Equivalentes

Tabla 6. Áreas experimentales y equivalentes en Córdoba.

\begin{tabular}{|c|c|c|c|c|c|c|c|}
\hline \multirow{2}{*}{ Programa } & Proyecto & Denominación & $\begin{array}{c}\text { Población } \\
\text { (hab.) }\end{array}$ & $\begin{array}{c}\text { Valor } \\
\text { IDUM } \\
\text { (quintil) }\end{array}$ & Área experimental & \multicolumn{2}{c|}{ Área equivalente } \\
\cline { 2 - 7 } & ARB & $\begin{array}{c}\text { S. Martín } \\
\text { de Porres }\end{array}$ & $\begin{array}{c}\text { S. Martín de } \\
\text { Porres }\end{array}$ & 6.137 & quintil 5 & Moblación & $\begin{array}{c}\text { Valor } \\
\text { IDUM } \\
\text { (hab.) }\end{array}$ \\
\hline ARB & $\begin{array}{c}\text { Ajerquintil) } \\
\text { Norte }\end{array}$ & $\begin{array}{c}\text { S. Lorenzo, St. } \\
\text { Marina, S. Andrés }\end{array}$ & 12.037 & $-0,93(3)$ & $\begin{array}{c}\text { La Magdalena, Cerro } \\
\text { de la Golondrina y } \\
\text { Ribera }\end{array}$ & 9.814 & $1,31(5)$ \\
\hline
\end{tabular}

Fuente: Elaboración propia.

Mapa 4. Áreas experimentales y equivalentes en Córdoba.

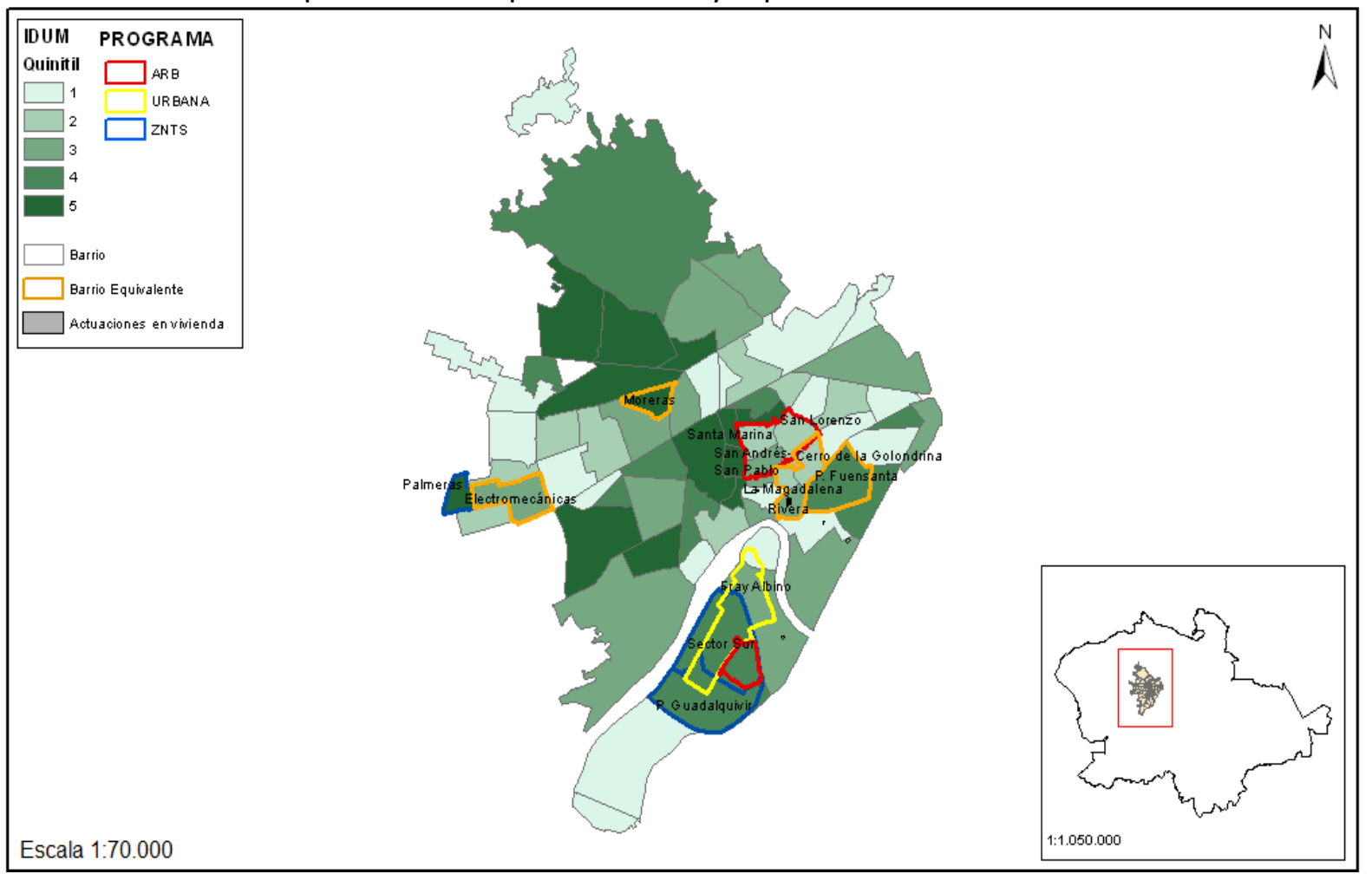

Fuente: Elaboración propia.

Revista de Estudios Andaluces, núm. 36 (2018) pp. 147-177. e-ISSN: 2340-2776

http://dx.doi.org/10.12795/rea.2018.i36.07

(c) $($ ) $\ominus$

Esta obra se distribuye con la licencia Creative Commons Reconocimiento-NoComercial-SinObraDerivada 4.0 Internacional (CC BY-NC-ND 4.0.) 
Tabla 7. Áreas experimentales y equivalentes en Dos Hermanas.

\begin{tabular}{|c|c|c|c|c|c|c|c|}
\hline \multirow{2}{*}{ Programa } & \multirow{2}{*}{ Proyecto } & \multicolumn{3}{|c|}{ Área experimental } & \multicolumn{3}{|c|}{ Área equivalente } \\
\hline & & Denominación & $\begin{array}{c}\text { Población } \\
\text { (hab.) }\end{array}$ & $\begin{array}{l}\text { Valor IDUM } \\
\text { (quintil) }\end{array}$ & Denominación & $\begin{array}{c}\text { Población } \\
\text { (hab.) }\end{array}$ & $\begin{array}{c}\text { Valor IDUM } \\
\text { (quintil) }\end{array}$ \\
\hline ZNTS & Ibarburu & Ibarburu & 5.058 & $1,6(5)$ & El Chaparral & 1.841 & $0,45(5)$ \\
\hline ZNTS & Los Montecillos & Los Montecillos & 4.322 & $-3,59(1)$ & Los Potros & 1.310 & $-3,62(1)$ \\
\hline
\end{tabular}

Fuente: Elaboración propia.

Mapa 5. Áreas experimentales y equivalentes en Dos Hermanas.

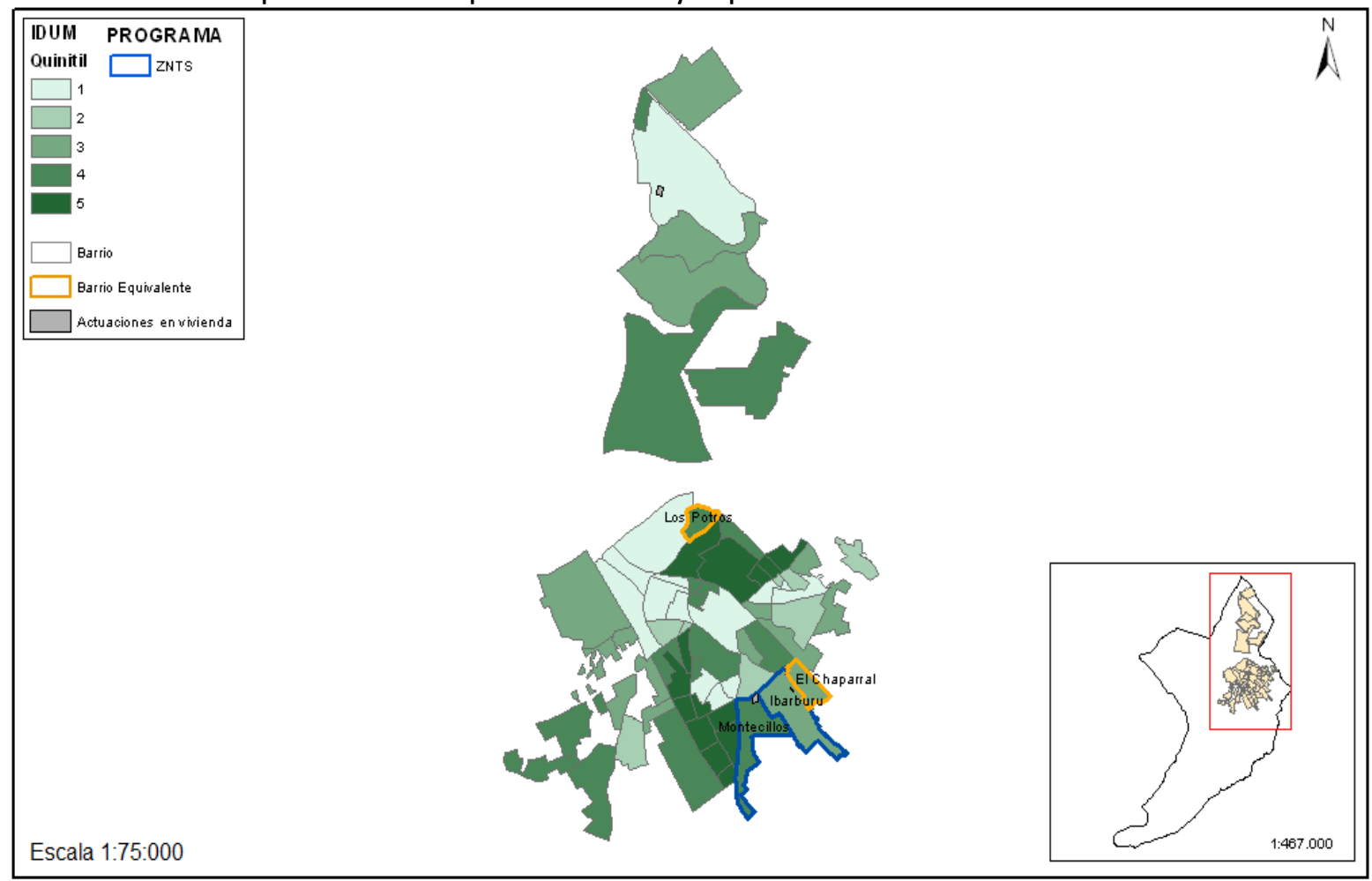

Fuente: Elaboración propia.

Revista de Estudios Andaluces, núm. 36 (2018) pp.147-177. e-ISSN: 2340-2776 http://dx.doi.org/10.12795/rea.2018.i36.07

\section{c) (i) $(9)$}


La Evaluación de las Políticas de Regeneración Urbana.

Propuesta Metodológica para la Delimitación de Áreas Experimentales y Equivalentes

Tabla 8. Áreas experimentales y equivalentes en Granada.

\begin{tabular}{|c|c|c|c|c|c|c|c|}
\hline \multirow[b]{2}{*}{ Programa } & \multirow[b]{2}{*}{ Proyecto } & \multicolumn{3}{|c|}{ Área experimental } & \multicolumn{3}{|c|}{ Área equivalente } \\
\hline & & Denominación & $\begin{array}{l}\text { Población } \\
\text { (hab.) }\end{array}$ & $\begin{array}{l}\text { Valor } \\
\text { IDUM } \\
\text { (quintil) }\end{array}$ & Denominación & $\begin{array}{l}\text { Población } \\
\text { (hab.) }\end{array}$ & $\begin{array}{l}\text { Valor } \\
\text { IDUM } \\
\text { (quintil) }\end{array}$ \\
\hline ARB & Bajo Albaicín & $\begin{array}{c}\text { secciones } 4002 \\
4003 \text { y } 7001\end{array}$ & 3.069 & $-0,38(4)$ & $\begin{array}{c}\text { secciones 5002, } \\
8001,8002 \\
8003,8004\end{array}$ & 5.028 & $-0,47(4)$ \\
\hline ARB & Almanjáyar & $\begin{array}{c}\text { Almanjáyar, } \\
\text { Cartuja, Rey Badis, } \\
\text { La Paz, Juaquina } \\
\text { Eguaras }\end{array}$ & 16.673 & $1,05(5)$ & $\begin{array}{l}\text { Cerrillo de } \\
\text { Maracena }\end{array}$ & 5.409 & $0,83(5)$ \\
\hline ZNTS & Distrito Norte I & Almanjáyar & 3.694 & $-0,98(2)$ & $\begin{array}{l}\text { S. Francisco } \\
\text { Javier }\end{array}$ & 3.519 & $-1,16(2)$ \\
\hline ZNTS & Distrito Norte II & Cartuja & 5.799 & $1,43(5)$ & $\begin{array}{l}\text { Cerrillo de } \\
\text { Maracena }\end{array}$ & 5.409 & $0,83(5)$ \\
\hline ZNTS & Distrito Norte III & Montijo & 2.828 & $-0,28(4)$ & $\begin{array}{c}\text { Parque Nueva } \\
\text { Granada }\end{array}$ & 3.050 & $-0,40(4)$ \\
\hline
\end{tabular}

Fuente: Elaboración propia.

Mapa 6. Áreas experimentales y equivalentes en Granada.

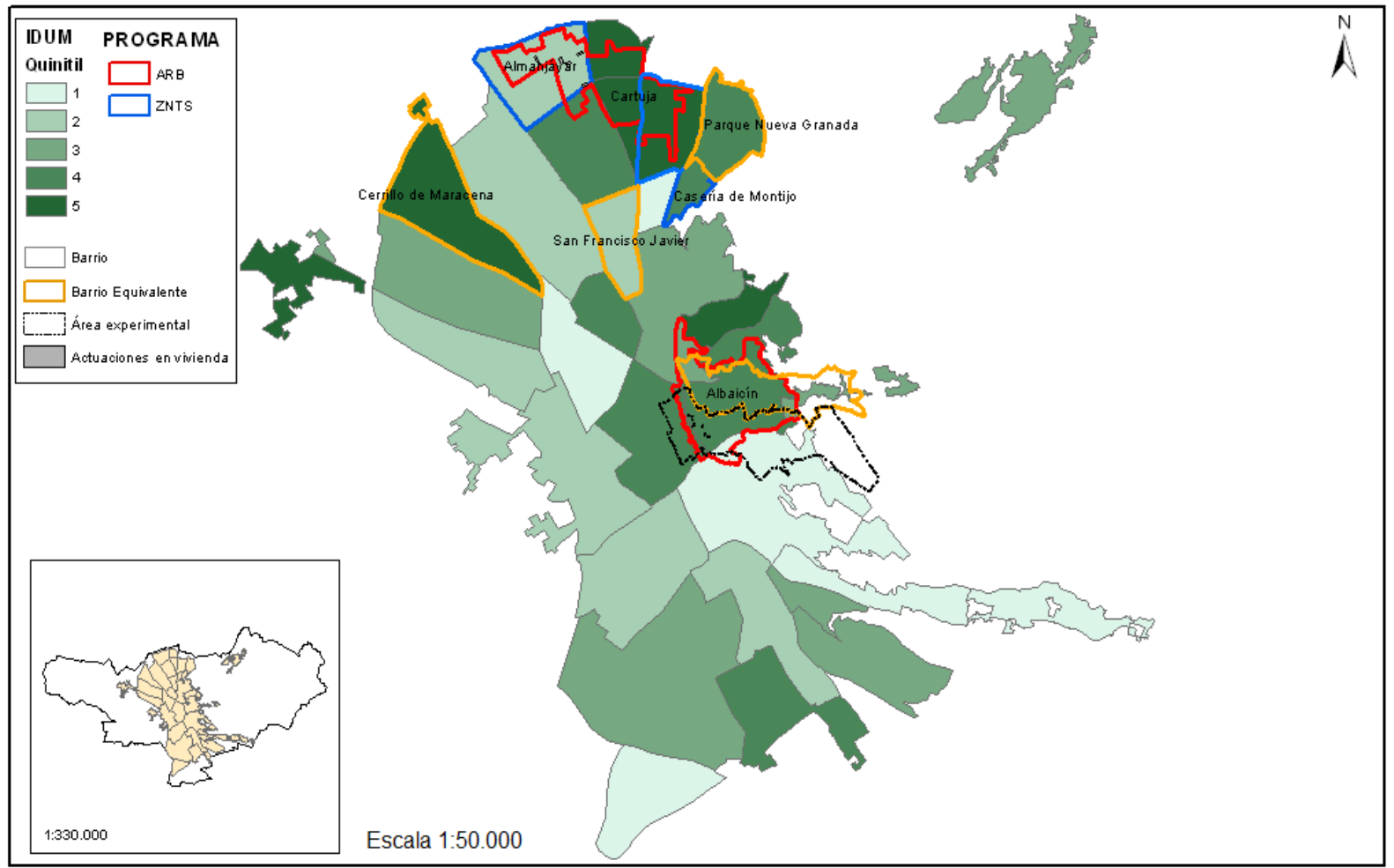

Fuente: Elaboración propia.

Revista de Estudios Andaluces, núm. 36 (2018) pp. 147-177. e-ISSN: 2340-2776

http://dx.doi.org/10.12795/rea.2018.i36.07

(c) (†) $\ominus$

Esta obra se distribuye con la licencia Creative Commons Reconocimiento-NoComercial-SinObraDerivada 4.0 Internacional (CC BY-NC-ND 4.0.) 
Tabla 9. Áreas experimentales y equivalentes en Huelva.

\begin{tabular}{|c|c|c|c|c|c|c|c|}
\hline \multirow{2}{*}{ Programa } & Proyecto & \multicolumn{4}{|c|}{ Área experimental } & \multicolumn{3}{c|}{ Área equivalente } \\
\cline { 3 - 8 } & Denominación & $\begin{array}{c}\text { Población } \\
\text { (hab.) }\end{array}$ & $\begin{array}{c}\text { Valor } \\
\text { IDUM } \\
\text { (quintil) }\end{array}$ & Denominación & $\begin{array}{c}\text { Población } \\
\text { (hab.) }\end{array}$ & $\begin{array}{c}\text { Valor } \\
\text { IDUM } \\
\text { (quintil) }\end{array}$ \\
\hline ARB/ZNTS & $\begin{array}{c}\text { Marismas } \\
\text { del Odiel }\end{array}$ & $\begin{array}{c}\text { secciones 02007, } \\
02008,08001\end{array}$ & 3.369 & $3,57(5)$ & Bda. del Carmen & 2.726 & $2,03(5)$ \\
\hline ZNTS & Distrito 5 & Torrejón & 2.453 & $1,98(5)$ & Bda. del Carmen & 2.726 & $2,03(5)$ \\
\hline ZNTS & Distrito 6 & Pérez Cubillas & 2.279 & $2,82(5)$ & $\begin{array}{c}\text { Bda. del Rocío, } \\
\text { Bda. de la Cinta }\end{array}$ & 2.186 & $0,61(4)$ \\
\hline
\end{tabular}

Fuente: Elaboración propia.

Mapa 7. Áreas experimentales y equivalentes en Huelva.

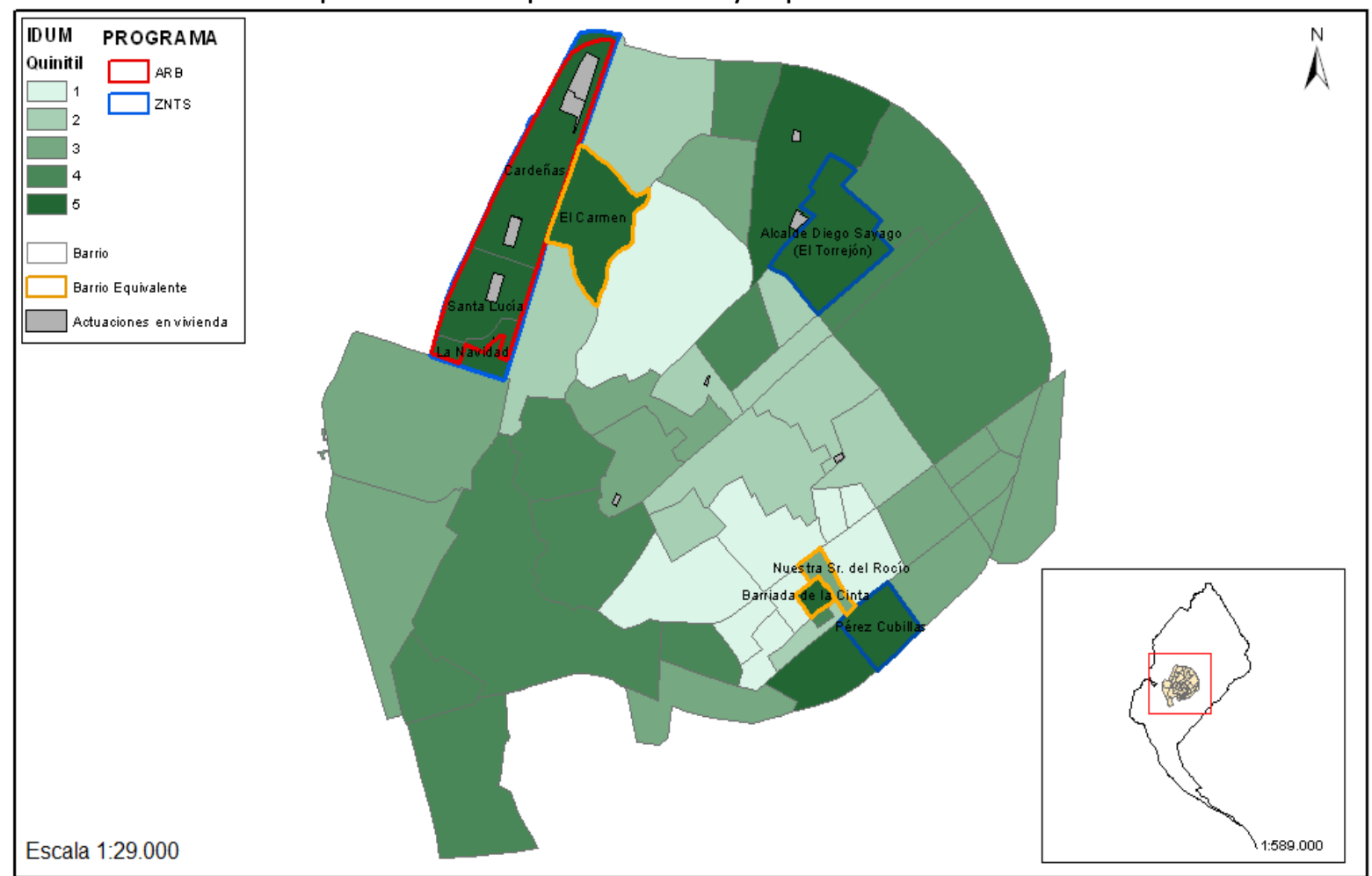

Fuente: Elaboración propia.

Revista de Estudios Andaluces, núm. 36 (2018) pp.147-177. e-ISSN: 2340-2776 http://dx.doi.org/10.12795/rea.2018.i36.07

\section{(c) (i) $\ominus$}


La Evaluación de las Políticas de Regeneración Urbana.

Propuesta Metodológica para la Delimitación de Áreas Experimentales y Equivalentes

Tabla 10. Áreas experimentales y equivalentes en Jaén.

\begin{tabular}{|c|c|c|c|c|c|c|}
\hline \multirow{2}{*}{ Proyecto } & \multicolumn{2}{|c|}{ Área experimental } & \multicolumn{2}{c|}{ Área equivalente } \\
\cline { 2 - 6 } & Denominación & $\begin{array}{c}\text { Población } \\
\text { (hab.) }\end{array}$ & $\begin{array}{c}\text { Valor } \\
\text { IDUM } \\
\text { (quintil) }\end{array}$ & $\begin{array}{c}\text { Denominación } \\
\text { Población } \\
\text { (hab.) }\end{array}$ & $\begin{array}{c}\text { Valor } \\
\text { IDUM } \\
\text { (quintil) }\end{array}$ \\
\hline $\begin{array}{c}\text { Centro } \\
\text { Histórico } \\
\text { de Jaén }\end{array}$ & $\begin{array}{c}\text { La Merced, S. Andrés, S. Juan, S. } \\
\text { Balomé, Sagrario, La Magdalena, } \\
\text { S. Vicente Paul, El Tomillo }\end{array}$ & 12.332 & quintil 3 & $\begin{array}{c}\text { Peñafecit- } \\
\text { Estación }\end{array}$ & 16.962 & $-0,77(3)$ \\
\hline $\begin{array}{c}\text { Plan } \\
\text { URBAN III } \\
\text { de Jaén }\end{array}$ & $\begin{array}{c}\text { Lartolomé, Sagrario, La Magdalena, } \\
\text { S. Vicente Paul, El Tomillo, El } \\
\text { Almendral, La Glorieta, S. Ildefonso- } \\
\text { La Alameda, S. Felipe }\end{array}$ & 23.404 & quintil 3 & $\begin{array}{c}\text { Peñafecit- } \\
\text { Estación }\end{array}$ & 16.962 & $-0,77$ (3) \\
\hline
\end{tabular}

Fuente: Elaboración propia.

Mapa 8. Áreas experimentales y equivalentes en Jaén.

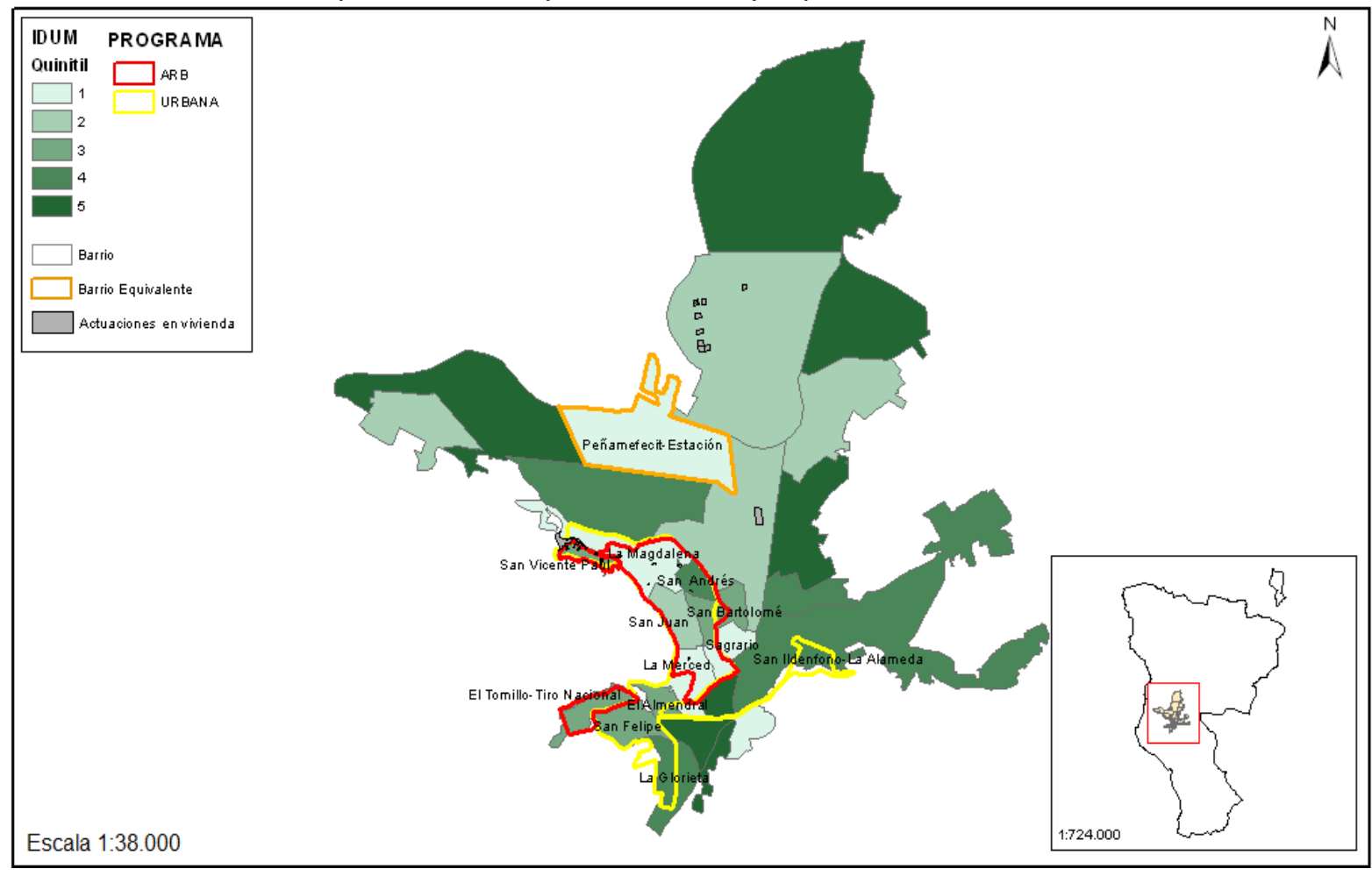

Fuente: Elaboración propia.

Revista de Estudios Andaluces, núm. 36 (2018) pp. 147-177. e-ISSN: 2340-2776

http://dx.doi.org/10.12795/rea.2018.i36.07

(c) $($ ) $(9)$

Esta obra se distribuye con la licencia Creative Commons Reconocimiento-NoComercial-SinObraDerivada 4.0 Internacional (CC BY-NC-ND 4.0.) 
Tabla 11. Áreas experimentales y equivalentes en Jerez de la Frontera.

\begin{tabular}{|c|c|c|c|c|c|c|c|}
\hline \multirow{2}{*}{ Programa } & \multirow{2}{*}{ Proyecto } & \multicolumn{3}{|c|}{ Área experimental } & \multicolumn{3}{c|}{ Área equivalente } \\
\cline { 3 - 8 } & Denominación & $\begin{array}{c}\text { Población } \\
\text { (hab.) }\end{array}$ & $\begin{array}{c}\text { Valor IDUM } \\
\text { (quintil) }\end{array}$ & Denominación & $\begin{array}{c}\text { Población } \\
\text { (hab.) }\end{array}$ & $\begin{array}{c}\text { Valor IDUM } \\
\text { (quintil) }\end{array}$ \\
\hline ARB & $\begin{array}{c}\text { Centro Hco. } \\
\text { de Jerez }\end{array}$ & Santiago & 2.896 & $-0,16(4)$ & Picadueñas & 2.841 & $-1,20(3)$ \\
\hline URBAN & $\begin{array}{c}\text { Zona Sur de } \\
\text { Jerez }\end{array}$ & S. Telmo Viejo & 3.424 & $1,6(5)$ & $\begin{array}{c}\text { S. Tomás de } \\
\text { Aquino }\end{array}$ & 1.362 & $1,09(4)$ \\
\hline ZNTS & Distrito Sur & Constitución & 1.521 & $-0,09(4)$ & $\begin{array}{c}\text { Barriada de } \\
\text { España }\end{array}$ & 2.037 & $-0,42(4)$ \\
\hline ZNTS & $\begin{array}{c}\text { Distrito } \\
\text { Oeste I }\end{array}$ & S. Juan de Dios & 6.699 & $-0,42(4)$ & Puente de Cádiz & 1.578 & $1,37(5)$ \\
\hline ZNTS & $\begin{array}{c}\text { Distrito } \\
\text { Oeste II }\end{array}$ & S. Benito & 1.711 & $2,08(5)$ & Puertas del Sur & 2.655 & $-0,35(4)$ \\
\hline
\end{tabular}

Fuente: Elaboración propia.

Mapa 9. Áreas experimentales y equivalentes en Jerez de la Frontera.

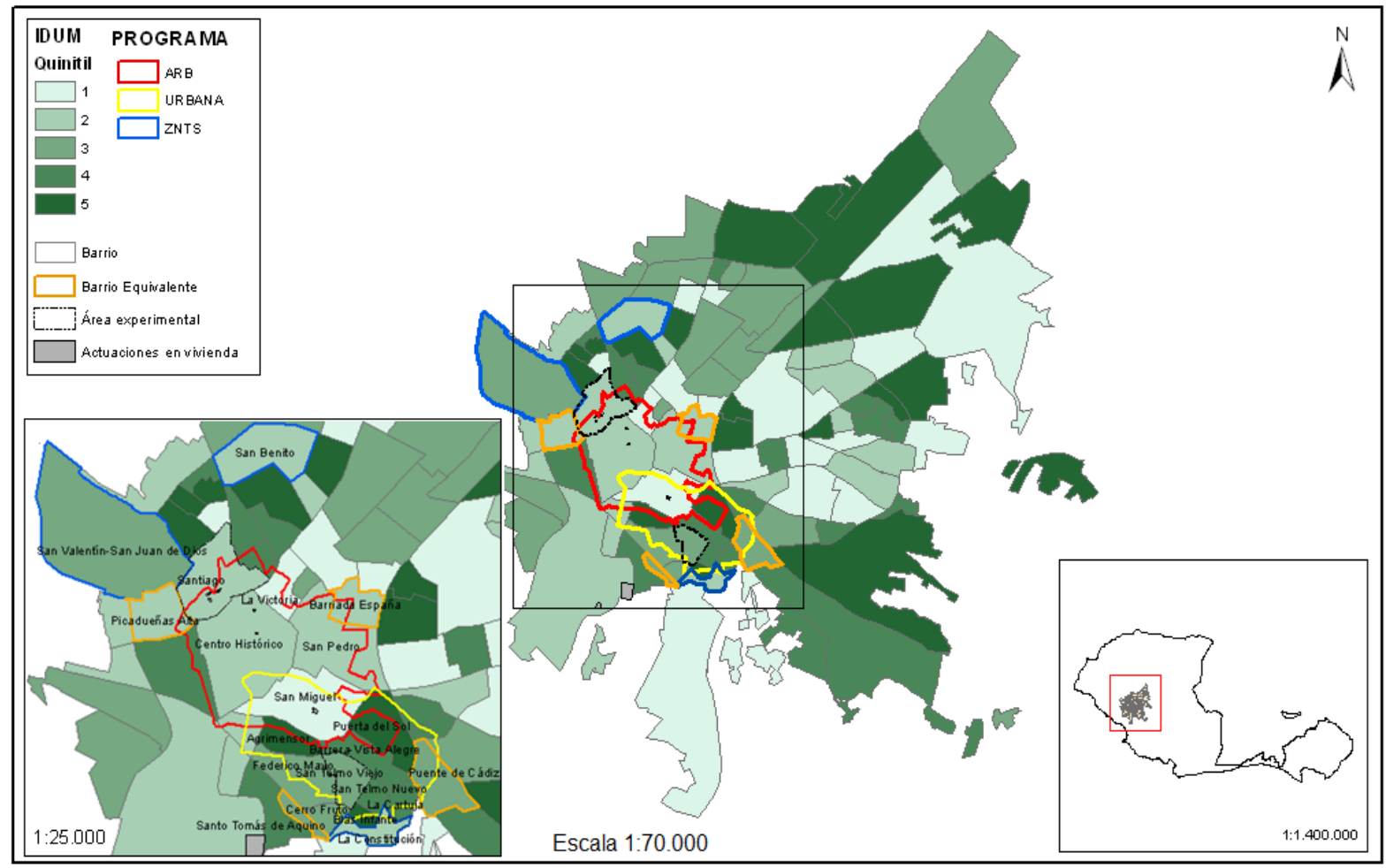

Fuente: Elaboración propia.

Revista de Estudios Andaluces, núm. 36 (2018) pp.147-177. e-ISSN: 2340-2776 http://dx.doi.org/10.12795/rea.2018.i36.07

\section{(c) (i) $\ominus$}


La Evaluación de las Políticas de Regeneración Urbana.

Propuesta Metodológica para la Delimitación de Áreas Experimentales y Equivalentes

Tabla 12. Áreas experimentales y equivalentes en Málaga.

\begin{tabular}{|c|c|c|c|c|c|c|c|}
\hline \multirow[b]{2}{*}{ Programa } & \multirow[b]{2}{*}{ Proyecto } & \multicolumn{3}{|c|}{ Área experimental } & \multicolumn{3}{|c|}{ Área equivalente } \\
\hline & & Denominación & $\begin{array}{c}\text { Población } \\
\text { (hab.) }\end{array}$ & $\begin{array}{c}\text { Valor } \\
\text { IDUM } \\
\text { (quintil) }\end{array}$ & Denominación & $\begin{array}{c}\text { Población } \\
\text { (hab.) }\end{array}$ & $\begin{array}{l}\text { Valor } \\
\text { IDUM } \\
\text { (quintil) }\end{array}$ \\
\hline ARB & $\begin{array}{l}\text { Casco Hco. } \\
\text { de Málaga }\end{array}$ & $\begin{array}{c}\text { Lagunilla, Las Victorias, La } \\
\text { Goleta, S. Felipe Neri, La } \\
\text { Merced }\end{array}$ & 7.833 & $0,24(3)$ & Trinidad Este & 9.538 & $-0,38(3)$ \\
\hline ARB & $\begin{array}{c}\text { Barriada } \\
\text { Las Flores }\end{array}$ & Barriada Las Flores & 2.759 & $-1,09(2)$ & La Roca & 3.377 & $-1,17(2)$ \\
\hline URBAN & $\begin{array}{l}\text { Arrabales } \\
\text { de Málaga }\end{array}$ & $\begin{array}{c}\text { La Victoria, Monte } \\
\text { Gibralfaro, Lagunillas, La } \\
\text { Goleta, Centro Hco., } \\
\text { Ensanche Centro, La } \\
\text { Merced, Capuchinos, S. } \\
\text { Felipe Neri }\end{array}$ & 21.581 & quintil 4 & $\begin{array}{l}\text { Trinidad Este y } \\
\text { Perchel Norte }\end{array}$ & 11.051 & $0,08(4)$ \\
\hline ZNTS & $\begin{array}{l}\text { Zona } \\
\text { Centro }\end{array}$ & Centro Histórico & 4.543 & quintil 4 & Perchel Norte & 4.695 & $-0,66(3)$ \\
\hline
\end{tabular}

Fuente: Elaboración propia.

Mapa 10. Áreas experimentales y equivalentes en Málaga.

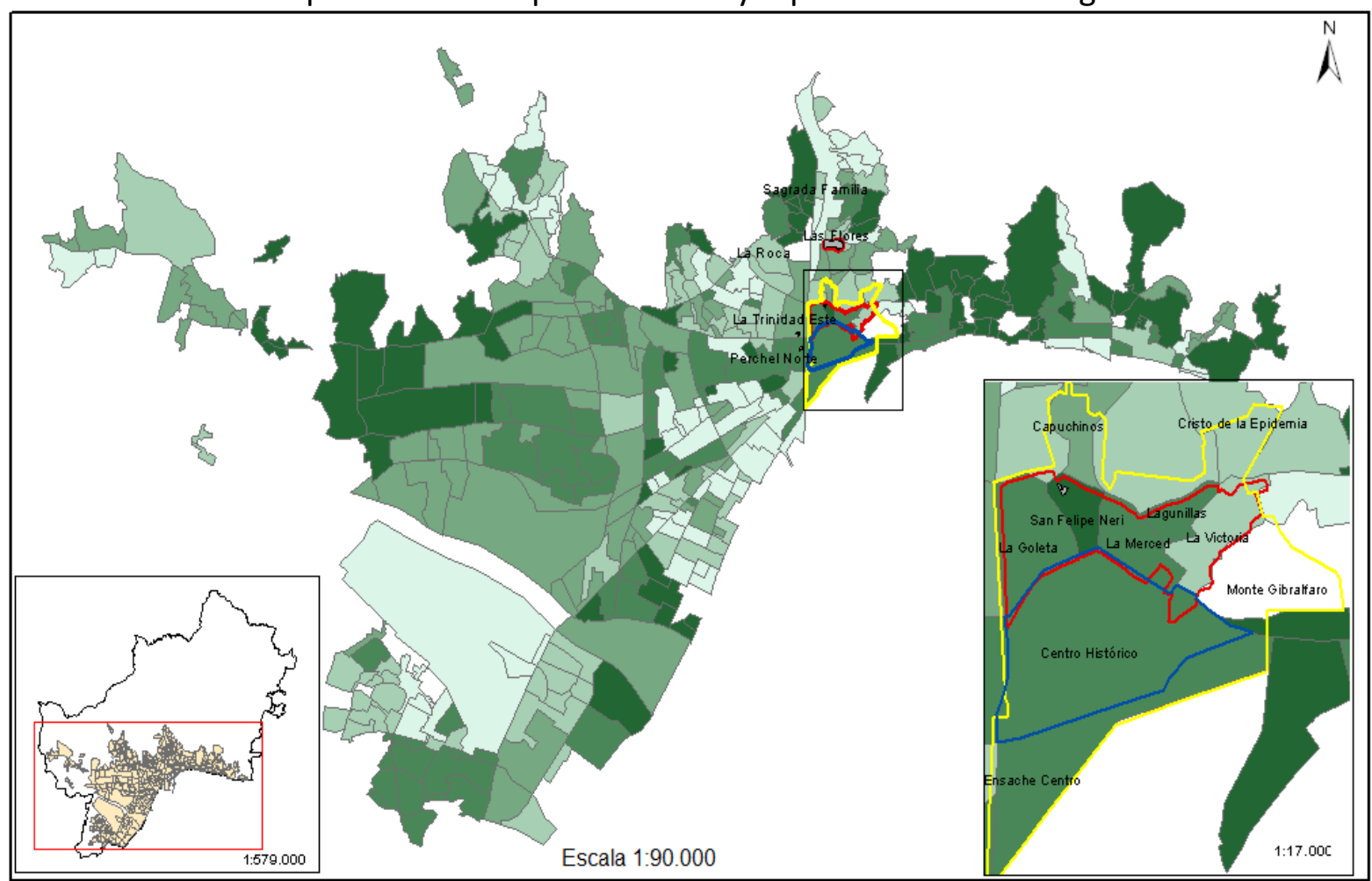

Fuente: Elaboración propia.

Revista de Estudios Andaluces, núm. 36 (2018) pp. 147-177. e-ISSN: 2340-2776

http://dx.doi.org/10.12795/rea.2018.i36.07

cc) (i) $\ominus$

Esta obra se distribuye con la licencia Creative Commons Reconocimiento-NoComercial-SinObraDerivada 4.0 Internacional (CC BY-NC-ND 4.0.) 
Tabla 13. Áreas experimentales y equivalentes en Sevilla.

\begin{tabular}{|c|c|c|c|c|c|c|c|}
\hline \multirow{2}{*}{ Programa } & \multirow{2}{*}{ Proyecto } & \multicolumn{3}{|c|}{ Área experimental } & \multicolumn{3}{c|}{ Área equivalente } \\
\cline { 3 - 8 } & Denominación & $\begin{array}{c}\text { Población } \\
\text { (hab.) }\end{array}$ & $\begin{array}{c}\text { Valor } \\
\text { IDUM } \\
\text { (quintil) }\end{array}$ & Denominación & $\begin{array}{c}\text { Población } \\
\text { (hab.) }\end{array}$ & $\begin{array}{c}\text { Valor } \\
\text { IDUM } \\
\text { (quintil) }\end{array}$ \\
\hline ARB/URBAN/ZNTS & Polígono Sur & $\begin{array}{c}\text { Oliva, Av. Paz, } \\
\text { Letanías y P. Sur. }\end{array}$ & 30.745 & quintil 5 & La Plata & 13.537 & $0,79(5)$ \\
\hline ZNTS & Casco Norte & $\begin{array}{c}\text { Feria, S. Julián y } \\
\text { S. Gil }\end{array}$ & 16.212 & $1,7(5)$ & $\begin{array}{c}\text { S. Bartolomé } \\
\text { Sta. Catalina }\end{array}$ & 7.516 & $3,00(5)$ \\
\hline ZNTS & Tres Barrios & $\begin{array}{c}\text { Los Pájaros, } \\
\text { Amate }\end{array}$ & 20.613 & $1,26(5)$ & $\begin{array}{c}\text { S. Jerónimo y } \\
\text { La Bachillera }\end{array}$ & 11.433 & $0,15(4)$ \\
\hline ZNTS & P. Norte & E. Norte & 6.910 & $1,48(5)$ & $\begin{array}{c}\text { Begoña-Sta. } \\
\text { Catalina }\end{array}$ & 3.212 & $0,65(5)$ \\
\hline ZNTS & Torreblanca & Torreblanca & 17.746 & $1,15(5)$ & Bellavista & 11.812 & $-0,17(4)$ \\
\hline
\end{tabular}

Fuente: Elaboración propia.

Mapa 11. Áreas experimentales y equivalentes en Sevilla.

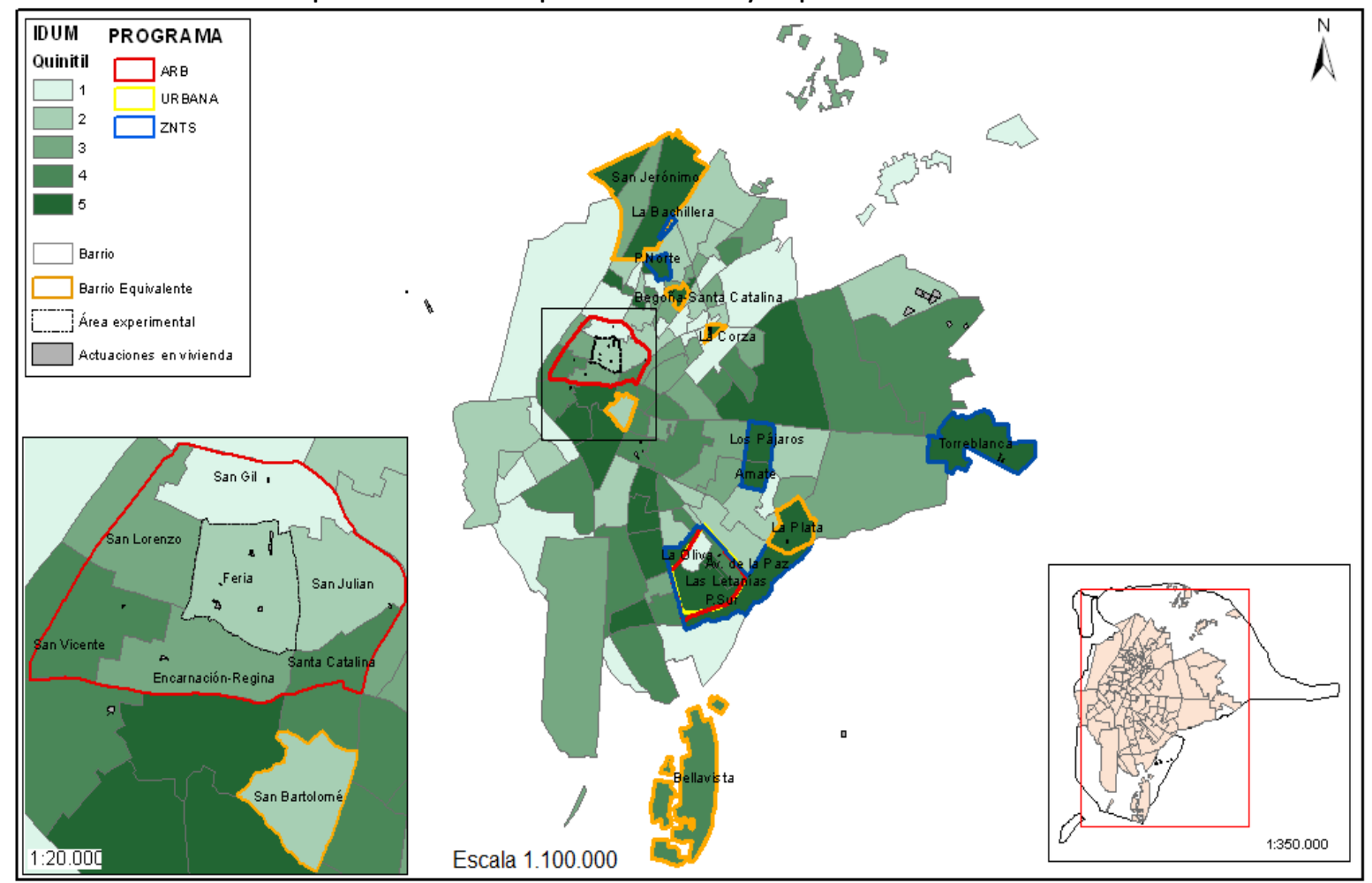

Fuente: Elaboración propia.

Revista de Estudios Andaluces, núm. 36 (2018) pp.147-177. e-ISSN: 2340-2776 http://dx.doi.org/10.12795/rea.2018.i36.07

\section{(c) (i) $\ominus$}




\section{VALIDACIÓN DE LOS RESULTADOS: SIMILITUDES Y DIFERENCIAS ENTRE ÁREAS EXPERIMENTALES Y EQUIVALENTES}

Una vez finalizado el proceso de delimitación de las áreas equivalentes, cabe plantearse la siguiente pregunta: ¿ ¿son las áreas experimentales y equivalentes similares en el momento previo al inicio de las intervenciones? Es condición necesaria para la posterior evaluación de los efectos de los programas de regeneración urbana tener la seguridad de que el cambio habido entre unas y otras no se debe a que ya tenían condiciones de partida muy diferentes. Por ello se requiere validar el ejercicio realizado.

Con el objeto de validar la selección de las áreas equivalentes se ha aplicado análisis de varianza, utilizando como variable independiente el hecho de que el área sea experimental o equivalente, y como variables dependientes algunas características socioeconómicas de las áreas a partir del Censo de Población y Vivienda del año 2001. Lo que tratamos de comprobar es que, a grandes rasgos, esas características son similares entre los dos tipos de áreas, y, por tanto, el análisis debe mostrar que las diferencias de medias entre ellas no son grandes, ni estadísticamente significativas (tomando como criterio de significatividad un valor de $p<0,05)$.

Tal y como se ha indicado más arriba, en algún caso se ha asignado una misma área equivalente para distintos proyectos (o áreas experimentales), por lo que no coincide exactamente el número total de áreas experimentales y el número total de áreas equivalentes. Además, para evitar duplicidad que pudiera afectar al análisis de datos se ha incluido un área experimental en el caso de existir superposición (esto es, aunque hay tres proyectos en Polígono Sur, en los análisis únicamente se considera un área experimental, que es la misma para los tres). Así, en su conjunto las unidades de observación (áreas territoriales) incluidas en los análisis son 43 áreas experimentales y 34 áreas equivalentes.

Los resultados muestran que, a grandes rasgos, existen bastantes similitudes entre áreas experimentales y áreas equivalentes (tal y como se puede apreciar en la tabla 14). De forma más detallada, puede apreciarse que son menos similares respecto a las variables o indicadores 'IDUM', personas analfabetas, tasa de paro; pero también respecto a la 'Condición socioeconómica media', dependencia juvenil, la 'antigüedad media de los edificios' y la percepción de delincuencia o vandalismo (en la columna de significatividad, el valor de $p<0,05$ ).

Esto puede suponer que existen algunos casos, algunas áreas experimentales, que destacan frente a las otras por presentar un mayor grado de vulnerabilidad urbana. Se trata de barrios o zonas de las ciudades que son bien conocidas al respecto, y que, por tanto, suponen un espacio territorial específico y muy diferente del resto. En concreto se trataría de las siguientes áreas experimentales (o proyectos): ARB y ZNTS 'Marismas del Odiel' y ZNTS 'Pérez Cubillas' en Huelva; ZNTS y los ARB/URBAN/ZNTS 'Polígono Sur' y ZNTS 'El Vacie' en Sevilla. No en vano, todas estas áreas se encuentran en el quinto quintil del indicador ÍDUM (máximo nivel de vulnerabilidad urbana); poseen el mayor porcentaje de población extranjera; mayor proporción de personas sin estudios; peores características del parque residencial (más antigüedad de las viviendas y edificios, menor tamaño, 
Tabla 14. Áreas experimentales y equivalentes: similitudes y diferencias en 2001(sin duplicados).

\begin{tabular}{|c|c|c|c|c|c|c|}
\hline \multirow{2}{*}{ Aspectos } & \multirow{2}{*}{ Indicador } & \multicolumn{3}{|c|}{ Medias } & \multirow{2}{*}{$\mathrm{F}$} & \multirow{2}{*}{ Sigf. } \\
\hline & & A. Exp. & A. Eq. & Total & & \\
\hline Vulnerabilidad & IDUM & 67 &,- 09 & ,34 & 4,56 & ,036 \\
\hline \multirow{4}{*}{ Demográficos } & Población & $9.198,35$ & $6.410,35$ & $7.967,29$ & 3,13 & 081 \\
\hline & Extranjeros no comunitarios (\%) & 2,05 & 1,10 & 1,63 & 2,86 & 095 \\
\hline & Indicador dependencia senil & ,34 & ,33 & ,33 & ,20 & ,655 \\
\hline & Indicador dependencia juvenil & 47 & 42 & ,44 & 4,50 & 037 \\
\hline \multirow{5}{*}{ Socioeconómicos } & Analfabetos & 59,64 & 43,90 & 52,69 & 4,04 & 048 \\
\hline & Sin Estudios & 183,47 & 162,22 & 174,08 & 1,48 & ,228 \\
\hline & Condición Socioeconómica media & ,74 & ,82 & ,77 & 9,29 & ,003 \\
\hline & Tasa de paro & 30,15 & 24,30 & 27,57 & 14,31 & 000 \\
\hline & Operarios sin cualificar (\%) & 20,55 & 17,50 & 19,20 & 1,19 & ,279 \\
\hline \multirow{7}{*}{ Vivienda } & Viviendas en alquiler (\%) & 21,82 & 15,74 & 19,13 & 1,83 & 180 \\
\hline & Viviendas vacías (\%) & 15,79 & 13,79 & 14,90 & 1,90 & 172 \\
\hline & Tamaño medio de la vivienda & 3,10 & 3,05 & 3,08 & ,28 & 600 \\
\hline & Antigüedad media de las viviendas (años) & 16,49 & 15,25 & 15,94 & 1,59 & ,212 \\
\hline & Antigüedad media de los edificios (años) & 38,72 & 31,58 & 35,57 & 4,92 & ,030 \\
\hline & Superficie útil vivienda/no miembros & 25,98 & 27,23 & 26,53 & 1,04 & ,312 \\
\hline & Viviendas sin servicio o aseo (\%) & 19,01 & 5,75 & 13,15 & 2,90 & 093 \\
\hline \multirow{5}{*}{ Entorno (1) } & Viviendas con delincuencia o vandalismo & 271,13 & 211,86 & 244,96 & 6,82 & 011 \\
\hline & Viviendas con malas comunicaciones & 89,45 & 74,82 & 82,99 & 1,10 & ,297 \\
\hline & Viviendas con poca limpieza en las calles & 219,20 & 195,08 & 208,55 & 1,98 & 163 \\
\hline & Viviendas con contaminación o malos olores & 130,41 & 117,66 & 124,78 & ,88 & 350 \\
\hline & Viviendas con ruidos exteriores & 198,02 & 182,20 & 191,03 & 1,39 & 243 \\
\hline
\end{tabular}

Fuente: Elaboración propia partir de Censo de Población y Vivienda (2001).

(1) Los indicadores del entorno se refiere a la percepción de sus residentes sobre cada uno de los problemas considerados.

Número de casos: Total=77, Experimentales=43, Equivalentes=34.

mayor densidad habitacional, más viviendas vacías) y los valores más altos en la percepción de problemas en el barrio (más delincuencia, peores comunicaciones, más ruidos, mayor grado de contaminación y malos olores; etc.). Se trata de casos extremos en la distribución de estas variables. Por ello, tras comprobar las singularidades de estas áreas, se procedió a repetir el análisis de varianza (ANOVA de un factor) sin incluir estos casos y sus respectivas áreas equivalentes, cuyos resultados se muestran en la tabla 15. Por tanto, los análisis suponen la existencia de 54 áreas (30 áreas experimentales y 24 equivalentes) para el conjunto de las ciudades andaluzas analizadas.

Revista de Estudios Andaluces, núm. 36 (2018) pp.147-177. e-ISSN: 2340-2776 http://dx.doi.org/10.12795/rea.2018.i36.07

(c) $($ ) $\odot \ominus$

cc) Esta obra se distribuye con la licencia Creative Commons Reconocimiento-NoComercial-SinObraDerivada 4.0 Internacional (CC BY-NC-ND 4.0.) 
Tabla 15. Áreas experimentales y equivalentes: similitudes y diferencias en 2001 (sin áreas con mayor grado de vulnerabilidad urbana).

\begin{tabular}{|c|c|c|c|c|c|c|}
\hline \multirow{2}{*}{ Aspectos } & \multirow{2}{*}{ Indicador } & \multicolumn{3}{|c|}{ Medias } & \multirow{2}{*}{$F$} & \multirow{2}{*}{ Sigf. } \\
\hline & & A. Exp. & A. Eq. & Total & & \\
\hline Vulnerabilidad & IDUM & ,00 &,- 33 &,- 15 & ,94 & ,336 \\
\hline \multirow{4}{*}{ Demográficos } & Población & 10.039 & 8.319 & 8.386 & 4,474 & 059 \\
\hline & Extranjeros no comunitarios (\%) & 1,86 & 1,20 & 1,56 & 1,772 & 189 \\
\hline & Indicador dependencia senil & ,36 & ,35 & ,35 & ,170 & ,682 \\
\hline & Indicador dependencia juvenil & ,439 & 397 & ,420 & 2,128 & 151 \\
\hline \multirow{5}{*}{ Socioeconómicos } & Analfabetos & 54,16 & 38,98 & 47,41 & 3,335 & 074 \\
\hline & Sin Estudios & 177,12 & 155,18 & 167,37 & 1,121 & ,295 \\
\hline & Condición Socioeconómica media & ,76 & ,84 & ,80 & 5,163 & 027 \\
\hline & Tasa de paro & 29,65 & 25,60 & 27,96 & 10,056 & ,003 \\
\hline & Operarios sin cualificar (\%) & 18,40 & 16,96 & 17,76 & 187 & ,668 \\
\hline \multirow{7}{*}{ Vivienda } & Viviendas en alquiler (\%) & 20,24 & 12,33 & 16,73 & 3,448 & ,069 \\
\hline & Viviendas vacías (\%) & 16,84 & 14,85 & 15,96 & 1,45 & ,23 \\
\hline & Tamaño medio de la vivienda & 3,03 & 3,01 & 3,02 & ,032 & ,858 \\
\hline & $\begin{array}{l}\text { Antigüedad media de las viviendas } \\
\text { (años) }\end{array}$ & 16,68 & 15,82 & 16,29 & 651 & 423 \\
\hline & $\begin{array}{l}\text { Antigüedad media de los edificios } \\
\text { (años) }\end{array}$ & 40,19 & 41,77 & 36,45 & 6,151 & ,056 \\
\hline & Superficie útil vivienda/no miembros & 27,49 & 28,15 & 27,78 & 180 & ,673 \\
\hline & Viviendas sin servicio o aseo (\%) & 7,02 & 5,01 & 6,13 & 1,574 & ,215 \\
\hline \multirow{5}{*}{ Entorno } & $\begin{array}{l}\text { Viviendas con delincuencia } 0 \\
\text { vandalismo }\end{array}$ & 267,72 & 219,93 & 246,48 & 3,022 & ,088 \\
\hline & Viviendas con malas comunicaciones & 79,24 & 81,65 & 80,31 & ,021 & ,886 \\
\hline & $\begin{array}{l}\text { Viviendas con poca limpieza en las } \\
\text { calles }\end{array}$ & 219,41 & 201,31 & 211,36 & ,773 & ,383 \\
\hline & $\begin{array}{l}\text { Viviendas con contaminación o malos } \\
\text { olores }\end{array}$ & 122,13 & 112,37 & 117,79 & ,482 & ,491 \\
\hline & Viviendas con ruidos exteriores & 194,81 & 184,16 & 190,08 & ,493 & ,486 \\
\hline
\end{tabular}

Fuente: Elaboración propia partir de Censo de Población y Vivienda (2001).

Número de casos: Total=54, Experimentales=30, Equivalentes $=24$.

Tal y como se puede apreciar en la tabla anterior los resultados muestran que las distribuciones de los dos tipos de áreas ya no difieren en cuanto al 'IDUM' o en la mayoría de los otros indicadores señalados más arriba; solamente persistiendo diferencias en cuanto a la 'Condición socioeconómica media' y la tasa de paro (aunque la diferencia no es muy grande, 29,6\% de tasa de paro en las áreas experimentales y $25 \%$ en las áreas equivalentes). En definitiva, y atendiendo a los resultados obtenidos, podemos afirmar que las áreas comparadas (experimentales y equivalentes) son similares en cuanto a características demográficas, socioeconómicas y características del parque residencial. 
Por otra parte, para validar la selección de las áreas también se ha procedido a comparar el número de actuaciones realizadas en el marco del programa ARB. Este supone un conjunto de medidas de mejora del entorno urbano que pueden realizarse en el conjunto de los municipios andaluces y sus áreas sub-municipales, pero que se concentran en las áreas experimentales. Para ello se elaboraron dos indicadores: ' $\mathrm{n}$ o de actuaciones por superficie (Ha)' y ' $\mathrm{n}$ o de actuaciones por cada 100 habitantes'. Tal y como puede apreciarse en la tabla siguiente las medias son siempre superiores para las áreas experimentales, siendo las diferencias con las áreas equivalentes estadísticamente significativas (para $p<0,05$ ), tanto si se consideran las actuaciones por superficie, como por habitantes. Por tanto, aunque sean similares en cuanto a sus rasgos socio-económicos y urbanos, se diferencian claramente en el hecho de que se produzca -o no- en ellas intervención mediante un proyecto de regeneración urbana.

Tabla 16. Densidad de actuaciones en áreas experimentales y equivalentes* en 2001.

\begin{tabular}{|c|c|c|c|c|c|c|}
\hline \multirow{2}{*}{ Aspectos } & \multirow{2}{*}{ Indicador } & \multicolumn{3}{|c|}{ Medias } & \multirow{2}{*}{$F$} & \multirow{2}{*}{ Sigf. } \\
\hline & & A. Exp. & A. Eq. & Total & & \\
\hline \multirow{2}{*}{$\begin{array}{l}\text { Densidad de } \\
\text { actuaciones }\end{array}$} & ACT_DIV-Ha & 2,75 & ,62 & 1,76 & 7,10 & 013 \\
\hline & ACT_DIV_POB2001 & 1,95 & ,36 & 1,21 & 22,33 & ,000 \\
\hline
\end{tabular}

*N=30: (16 exp. y 14 eq.). En este caso se ha reducido el tamaño $\mathrm{N}$ debido a que los indicadores se han elaborado solamente con las actuaciones del programa ARB.

Fuente: Elaboración propia partir de Censo de Población y Vivienda (2001)

Número de casos: Total=30, Experimentales=16, Equivalentes=14.

\section{CONCLUSIONES}

Tras definir qué es un área experimental y qué un área equivalente, proceder a su delimitación mediante una serie de criterios de diversa índole; que van desde la mera morfología urbana hasta aspectos que tienen que ver con la localización (centro-periferia), la densidad de actuaciones, pasando por criterios demográficos y socioeconómicos, hemos pasado a contestar a la siguiente pregunta: ¿eran similares las áreas experimentales y equivalentes al iniciarse en las primeras los proyectos de intervención? Tras la eliminación en una segunda validación de los resultados de los casos extremos, esto es, las áreas de mayor vulnerabilidad urbana, parecen existir similitudes entre las áreas comparadas (experimentales y equivalentes) en los criterios básicos utilizados para definirlas, además de mostrarse consistentes en todos los análisis las diferencias en cuanto a la densidad de actuaciones.

En la gran mayoría de las ocasiones se han obtenido áreas equivalentes cumpliendo los requisitos planteados, pero en otras, y debido a la mayor complejidad en la definición del proyecto original, es necesario aplicar criterios ad-hoc para la obtención de dichas equivalencias, derivados de la propia definición y características de algunas áreas experimentales, lo que muestran que ya, entre ellas, existe cierta heterogeneidad.

A esto debe unirse que, en algunos casos, tal delimitación ha debido adaptarse a la disponibilidad de información que ofrece el Censo de Población y Viviendas de 2001, que impone severas limitaciones para el desarrollo del análisis al nivel de desagregación territorial para secciones Revista de Estudios Andaluces, núm. 36 (2018) pp.147-177. e-ISSN: 2340-2776 http://dx.doi.org/10.12795/rea.2018.i36.07

(c) (†) $\ominus$

(c) Esta obra se distribuye con la licencia Creative Commons Reconocimiento-NoComercial-SinObraDerivada 4.0 Internacional (CC BY-NC-ND 4.0.) 
censales y su agrupación en barrios. Queda para posteriores trabajos la realización de análisis con datos del Censo de Población y Viviendas de 2011, una vez que han sido ejecutados los proyectos de regeneración urbana, que permitirá ver la evolución de las áreas experimentales y equivalentes y establecer comparaciones entre ambas y con el conjunto de la ciudad.

En resumen, tras los análisis realizados cabe afirmar que las áreas equivalentes son similares, en cuanto a características demográficas, socioeconómicas y características del parque residencial, a las áreas experimentales. No obstante, debe señalarse que la metodología planteada de búsqueda de pares semejantes presenta dificultades para su aplicación en casos extremos (barrios más vulnerables). Dicho de otra manera, existen barrios que por sus características son difícilmente comparables con ningún otro, por lo que debe considerarse este hecho en los análisis evaluativos.

En definitiva, esta metodología se presenta como un primer paso para, a posteriori, medir los efectos de la política pública de regeneración urbana que permita una evaluación objetiva de los resultados de los proyectos ejecutados mediante la selección de pares semejantes.

\section{REFERENCIAS}

Brama, A. (2013). The Effects of Neighbourhood Regeneration on the Neighbourhood Hierarchy of the Cities. En Van Ham et al. (eds.), Understanding Neighbourhood Dynamics (pp.111-138). New York: Springer.

Clark, E. (2005). The order and simplicity of gentrification: a political challenge. En Atkinson, R. y Bridge, G. (org.), Gentrification in a Global Context: the new urban colonialism. Oxon, Routledge. doi: https://doi.org/10.4324/9780203392089_chapter_16

Cordero Martín, G. (2000). Los servicios sociales en Andalucía (pp. 272-293). En Cano García, G. (dir.) Conocer Andalucía. Gran enciclopedia andaluza del siglo XXI. Vol.7.

Díaz Parra, I. (2013). La gentrificación en la cambiante estructura socioespacial de la ciudad. Biblio 3W. Revista Bibliográfica de Geografía y Ciencias Sociales. [En línea]. Universidad de Barcelona, Vol. XVIII, no 1030. Recuperado de <http://www.ub.es/geocrit/b3w-1030.htm>.

Glass, R. (1964). London, Aspects of Change. Londres: Macgibbon \& Kee.

Hamel, J. (1993). Case Study Methods. Newbury Park, Cal., Sage. doi: https://doi.org/10.4135/9781412983587

Hollister, R.G. y Hill, J. (1995). Problems in the evaluation of community-wide initiatives. En Connell, J.P., Kubisch, A.C. y Schorr, L.B. y Weiss, C.H. (eds.), New Approaches to Evaluating Community Initiatives (pp. 127-172). Washington, DC: Aspen Institute. 
Lawless, P. (2007). Continuing dilemmas for area based urban regeneration: evidence from the New Deal for Communities Programme in England. People, Place and Policy, 1(1), pp. 14-21. doi: https://doi.org/10.3351/ppp.0001.0001.0003

Ministerio de Fomento (2015). Atlas de Vulnerabilidad Urbana. [En línea] Recuperado de < http://atlasvulnerabilidadurbana.fomento.es/>

Navarro, C.J. (2013). Have creative cities a dark side? Cities, 35, pp. 213-220.

Navarro, C.J. y Rodríguez-García, M.J. (2015). Model of local governing coalitions: politics or policy effects? Urban Geography. doi: https://doi.org/10.1080/02723638.2015.1034485

O'Reilly, D. (2007). Comment on Rhodes et al. (2005): "Some further thoughts on assessing the effects of area-based initiatives on local outcomes". Urban Studies, 44(5/6), pp. 1145-1153. doi: https://doi.org/10.1080/00420980701255973

Rhodes, J., Tyler, P. y Brennan, A. (2005). Assessing the Effect of Area Based Initiatives on Local Area Outcomes: Some Thoughts Based on the National Evaluation of the Single Regeneration Budget in England. Urban Studies, 42(11), pp. 1919-1946. doi: https://doi.org/10.1080/00420980500280347

Rossi, P.H. (1999). Evaluating Community Development Programs: Problems and Prospects (pp. 521568). En Ferguson, R.F. y Dickens, W.T. (eds.): Urban Problems and Community Development, Washington, DC: The Brookings Institution.

Smelser, N.J. (1982). La comparazione nelle scienze social. Bologna, il Mulino.

Taylor, D. y Balloch, S. (2005). The politics of evaluation: an overview. En Taylor, D. and Balloch, S. (eds), The politics of evaluation, (pp.2-19). Bristol: The Polity Press.

Tobler, W. (1979). Cellular geography. En Gale, S. y G. Olsson (eds.), Philosophy in Geography, (Dordrecht: Reidel). doi: https://doi.org/10.1007/978-94-009-9394-5_18

Thomson, H. (2008). A dose of realism for healthy urban policy: lessons from area-based initiatives in UK. Journal of Epidemiological Community Health, 62, pp. 932-936. doi: https://doi.org/10.1136/jech.2007.068775

Wei, F. y Knox, P.L. (2014). Neighborhood Change in Metropolitan America, 1990 to 2010. Urban Affairs Review, 50(4), pp. 459-489. doi: https://doi.org/10.1177/1078087413501640

Revista de Estudios Andaluces, núm. 36 (2018) pp.147-177. e-ISSN: 2340-2776 http://dx.doi.org/10.12795/rea.2018.i36.07 